\title{
Theoretical Study of
}

\section{N-Methyl-3-Phenyl-3-(4-(Trifluoromethyl) Phenoxy) Propan as a Drug and Its Five Derivatives}

\author{
Qabas M. Abdul Hussein AL-Makhzumi*, Hussein I. Abdullah, Ramzie R. AL-Ani \\ Chemistry Department, College of Science, Mustansiriyah University, Baghdad, Iraq \\ Email: *qa bas.mh55@yahoo.com
}

How to cite this paper: AL-Makhzumi, Q.M.A.H., Abdullah, H.I. and AL-Ani, R.R. (2018) Theoretical Study of N-Methyl3-Phenyl-3-(4-(Trifluoromethyl)Phenoxy) Propan as a Drug and Its Five Derivatives. Journal of Biosciences and Medicines, 6 , 80-98.

https://doi.org/10.4236/jbm.2018.68007

Received: July 13, 2018

Accepted: August 26, 2018

Published: August 29, 2018

Copyright (c) 2018 by authors and Scientific Research Publishing Inc. This work is licensed under the Creative Commons Attribution International License (CC BY 4.0).

http://creativecommons.org/licenses/by/4.0/

\begin{abstract}
Quantum chemical calculation was correlated with geometrical structure and total energy of fluoxetine and its five derivatives. Theoretical vibrational frequencies and geometric parameters (bond lengths and bond angles) have been calculated using ab initio (HF), density functional theory (B3LYP), semi-empirical (AM1, PM3) methods with different basis sets to design the fluoxetine drugs and its derivatives by a Gaussian $09 \mathrm{~W}$ program. Theoretical optimized geometric parameters and vibrational frequencies of fluoxetine have been compared with the corresponding five derivatives data. The highest occupied molecular orbital (HOMO) and lowest unoccupied molecular orbital (LUMO) energies have been determined. The theoretical study includes the calculation of the thermodynamic properties of the drugs and its derivatives like zero-point energy, enthalpy, entropy, ionization energy, electron affinity to make a correlation between the gained results. The results of the four methods were not very clear, but an correlation between the dipole moment (potential character), static distribution (an active site character) and HOMO-LUMO energies (energy for electron transfer) shows that the patent 1.5 was important derivatives as a recommended drug relative to fluoxetine drug.
\end{abstract}

\section{Keywords}

Fluoxetine, AM1, PM3, DFT and HF: Thermodynamic Properties

\section{Introduction}

Fluoxetine hydrochloride $\left(\mathrm{C}_{17} \mathrm{H}_{18} \mathrm{~F}_{3} \mathrm{NO}\right)$ (Figure 1) is the first agent of the class of antidepressants known as selective serotonin-reuptake inhibitors (SSRIs). 
Fluoxetine

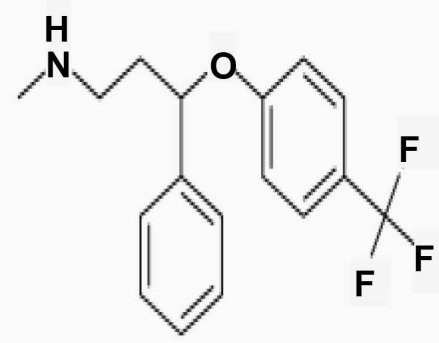

Patent 3

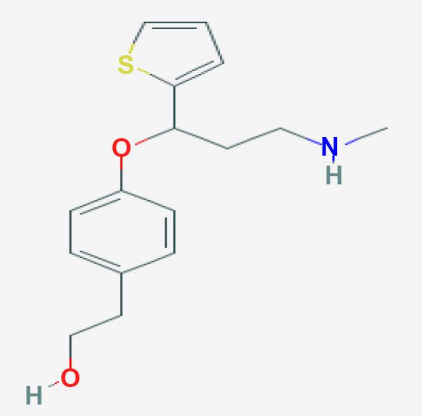

\section{Patent 1}

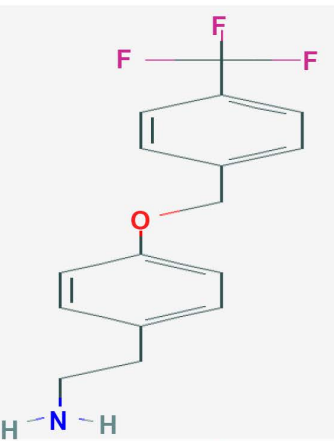

Patent 4

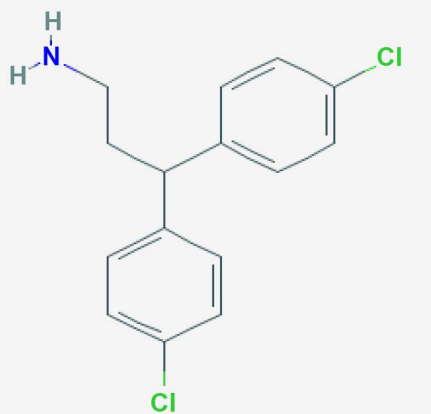

Patent 2

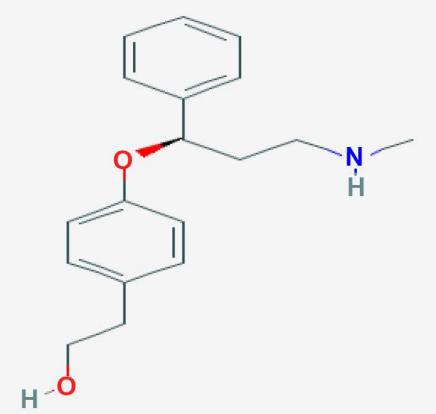

Patent 5

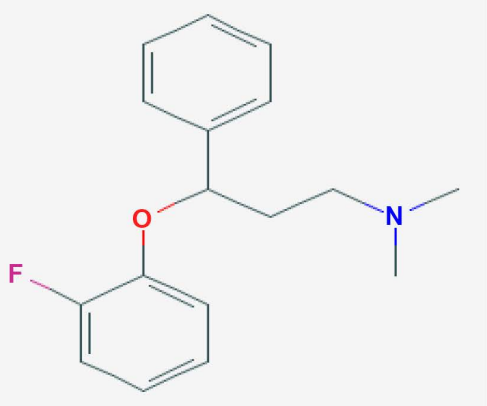

Figure 1. Structure of fluoxetine drugs and its derivatives.

Fluoxetine is a racemic mixture of the R- and S-enantiomers and are of equivalent pharmacologic activity. Despite distinct structural differences between compounds in this class, SSRIs possess similar pharmacological activity, as with other antidepressant agents [1]. The overall clinical effect of increased mood and decreased anxiety is thought to be due to adaptive changes in neuronal function that leads to enhanced serotonergic neurotransmission. Side effects include dry mouth, nausea, dizziness, drowsiness, sexual dysfunction and headache. Side effects generally occur within the first two weeks of therapy and are usually less severe and frequent than those observed with tricyclic antidepressants [1] [2]. Employed in the present work, calculations were used to determine the spectroscopic and electronic characters of the drug and its derivatives which are used to make a correlation in bioactivity future of them. It is important to identify the appropriate structures and the detailed electronic charge distribution; dipole moment, total energy and other properties in fluoxetine and its derivatives. Amide bonds are indeed present in a huge array of molecules, including major marketed drugs [3] [4] [5]. Hence amides and their derivatives have attracted continuing interest over the years. The five fluoxetine derivatives were prepared previously, but its bioactivity has not been characterized yet.

\section{Materials and Method}

The molecular structures of fluoxetine and its derivatives are presented in Table 1 and Figure 2 shows the structural formula, and the atomic position numbers assigned in this work. 
Q. M. A. H. AL-Makhzumi et al.

Table 1. Fluoxetine data with its derivatives.

\begin{tabular}{cccc}
\hline \multirow{2}{*}{ Name } & Formula & Molecular Weight & IUPAC Name \\
\cline { 2 - 4 } Fluoxetine & $\mathrm{C}_{17} \mathrm{H}_{18} \mathrm{~F}_{3} \mathrm{NO}$ & $309.332 \mathrm{~g} / \mathrm{mol}$ & N-methyl-3-phenyl-3-(4-(trifluoromethyl)phenoxy)propan \\
Patent 1 & $\mathrm{C}_{16} \mathrm{H}_{16} \mathrm{~F}_{3} \mathrm{NO}$ & $295.305 \mathrm{~g} / \mathrm{mol}$ & 2-[4-[[4-(trifluoromethyl)phenyl]methoxy]phenyl] ethanamine \\
Patent 2 & $\mathrm{C}_{18} \mathrm{H}_{23} \mathrm{NO}$ & $285.387 \mathrm{~g} / \mathrm{mol}$ & 2-[4-[(IS)-3-(methylamino)-1-phenylpropoxy]phenyl] ethanol \\
Patent 3 & $\mathrm{C}_{16} \mathrm{H}_{21} \mathrm{NO}_{2} \mathrm{~S}$ & $291.409 \mathrm{~g} / \mathrm{mol}$ & 2-[4-[(1S)-3-(methylamino)-1-thiophen-2-ylpropoxyl]phenyl]ethanol \\
Patent 4 & $\mathrm{C}_{15} \mathrm{H}_{15} \mathrm{Cl}_{2} \mathrm{~N}$ & $280.192 \mathrm{~g} / \mathrm{mol}$ & 3.3-bis(4-chlorophenyl)propan-1-amine \\
Patent 5 & $\mathrm{C}_{17} \mathrm{H}_{20} \mathrm{FNO}$ & $273.351 \mathrm{~g} / \mathrm{mol}$ & 3-(2-fluorophenoxy)-N,N-dimethyl-3-phenylpropan-1-amine \\
\hline
\end{tabular}

\section{Fluoxetine}

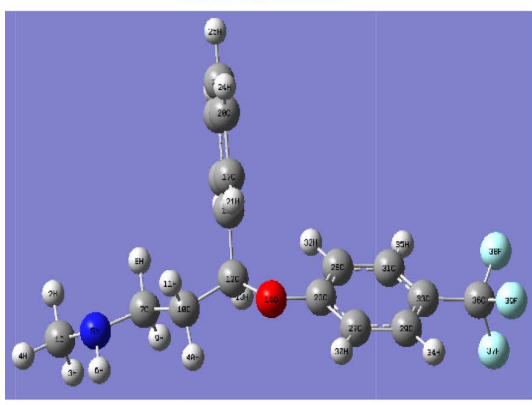

Patents 3

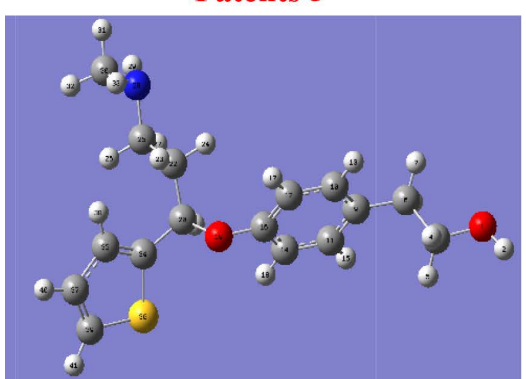

Patents 1

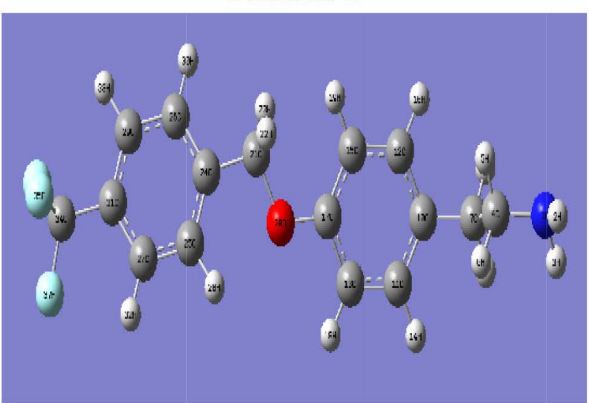

Patents 4

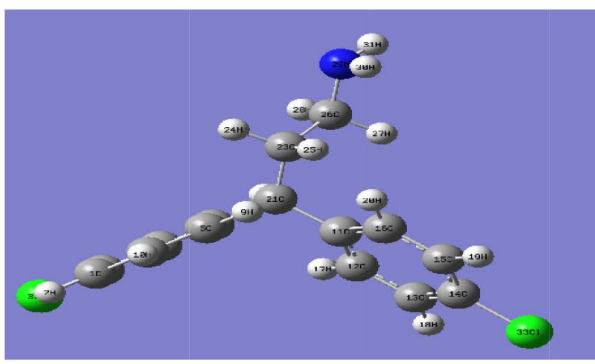

Patents 2

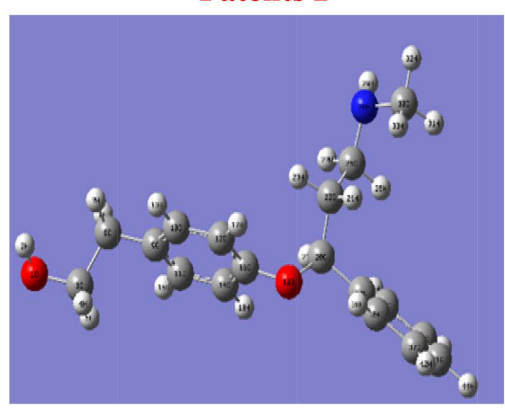

Patents 5

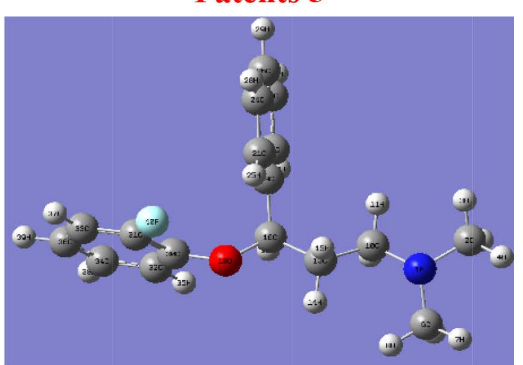

Figure 2. 3D structure of fluoxetine and its derivatives using Gaussian 09 program.

The program that used in the search.

\subsection{Gaussian 09}

Gaussian 09W (G09) is a computational chemistry program that runs on any modern windows 32 bit PC, or on a 64 bit PC [6]. An electronic structure package capable of predicting many properties of atoms, molecules, reactive system, e.g.:

- Molecular energies;

- Structure;

- Vibrational frequencies;

- Utilizing an initio, density functional theory, semi-empirical.

* semi-empirical methods $\left(\mathrm{AM}_{1}, \mathrm{PM}_{3}, \mathrm{PM}_{6}, \mathrm{MNDO}\right)$;

* ab initio methods (HF, $\mathrm{MP}_{2}, \mathrm{CCSD}, \mathrm{QCISD}$ );

* density functional theory ( $\mathrm{B}_{3} \mathrm{LYP}, \mathrm{MO}_{6}$, Custom). 


\subsection{Gauss View 5.08}

- Graphical interface for Gaussian 09 [7] [8];

- Sketch molecules;

- Setup Gaussian 09 input files;

- Graphically examine results.

\section{Computational Details}

In the first step of the calculation, geometrical parameters of these structures were further optimized by using density functional theory DFT (B3LYP)/6-31G, HF, AM1, PM3 methods. On the basis of the lowest energy conformer, the bond length and bond angles were obtained using the four methods.

The electronic properties: HOMO-LUMO energies are calculated by four methods, based on the optimized structure for soluble in water solvent [9] [10].

Thermodynamic properties of the title compound at $310 \mathrm{k}$ temperature have been calculated using four methods. Moreover, the dipole moment and Mulliken atomic charge have also been studied using Gaussian $09 \mathrm{~W}$ program package.

The initial atomic coordinates for geometry optimization was taken from Gauss View software database [11]. The molecular structure of drug in the ground state (in water) was optimized by HF and DFT/B3LYP with AM1 and PM3 methods for basis set levels. The optimized structure of the molecule was used to calculate the vibrational frequency at four methods. The calculated thermal correction to energy was scaled by $219.57 \mathrm{Kcal} / \mathrm{mole}^{-1}$ (AM1), $213.34 \mathrm{Kcal} / \mathrm{mole}^{-1}$ (PM3), $230.67 \mathrm{Kcal} / \mathrm{mole}^{-1}\left(\mathrm{HF}\right.$ ), and $216.51 \mathrm{Kcal} / \mathrm{mole}^{-1}$ (DFT) for fluoxetine and compare with the thermal correction energy for five derivatives.

\section{Result and Discussion}

\subsection{Molecular Structure}

The schematic depiction of fluoxetine and optimized structure are shown in Figure 2 and optimized bond lengths, bond angles of fluoxetine molecule which were calculated by using HF, DFT, AM1, PM3 methods with different basis sets are shown in Tables 2-5 compares the calculated geometric parameters with the five derivatives data. Based on this comparison, the bond length, angles for the derivatives showed good agreement with the drugs. From the theoretical values, we can find that most of the optimized bond lengths and bond angles for drug its slightly alike with the derivatives values, comparing bond angles and length of B3LYP with those of H, DFT and AM1, PM3. In spite of the differences, calculated geometric parameters represent a good approximation and they are the best for calculating other parameters, such as vibrational frequencies and thermodynamic properties.

\subsection{Thermodynamic Parameters and Molecular Properties}

To evaluate the energetic behavior of the title compound in water solvent media theoretical calculations were carried out at $310 \mathrm{k}$. Total energies and dipole 
Q. M. A. H. AL-Makhzumi et al.

Table 2. Selected bond distances $(\AA)$ bond lengths for fluoxetine and its five derivatives.

\begin{tabular}{|c|c|c|c|c|c|c|c|c|c|c|c|c|}
\hline \multirow{3}{*}{ Bond } & \multicolumn{12}{|c|}{ Bond Length (G09) } \\
\hline & \multicolumn{2}{|c|}{ Fluoxetine } & \multicolumn{2}{|c|}{ Patent 1} & \multicolumn{2}{|c|}{ Patent 2} & \multicolumn{2}{|c|}{ Patent 3} & \multicolumn{2}{|c|}{ Patent 4} & \multicolumn{2}{|c|}{ Patent 5} \\
\hline & AM1 & PM3 & AM1 & PM3 & AM1 & PM3 & AM1 & PM3 & AM1 & PM3 & AM1 & PM3 \\
\hline $\mathrm{C}_{1}-\mathrm{N}$ & 1.44 & 1.47 & 1.45 & 1.48 & 1.44 & 1.47 & 1.44 & 1.47 & 1.44 & 1.48 & 1.45 & 1.48 \\
\hline $\mathrm{N}-\mathrm{C}_{7}$ & 1.45 & 1.48 & $\cdots \cdots$ & $\cdots \cdots$ & 1.45 & 1.48 & 1.45 & 1.48 & $\cdots \cdots$ & & 1.46 & 1.49 \\
\hline $\mathrm{C}_{12}-\mathrm{O}_{14}$ & 1.44 & 1.44 & 1.43 & 1.41 & 1.44 & 1.48 & 1.45 & 1.44 & $\cdots \cdots$ & $\ldots$ & 1.44 & 1.43 \\
\hline $\mathrm{O}_{14}-\mathrm{C}_{26}$ & 1.37 & 1.37 & 1.38 & 1.38 & 1.38 & 1.38 & 1.39 & 1.39 & $\cdots \cdots$ & $\cdots$ & 1.38 & 1.37 \\
\hline $\mathrm{C}_{10}-\mathrm{C}_{12}$ & 1.53 & 1.53 & 1.53 & 1.50 & 1.52 & 1.52 & 1.53 & 1.52 & 1.53 & 1.52 & 1.52 & 1.52 \\
\hline $\mathrm{C}_{7}-\mathrm{C}_{10}$ & 1.52 & 1.52 & 1.52 & 1.52 & 1.53 & 1.53 & 1.53 & 1.54 & 1.52 & 1.53 & 1.53 & 1.53 \\
\hline C-F & 1.37 & 1.36 & 1.37 & 1.35 & $\ldots$. & ..... & ........ & ....... & ....... & $\ldots$ & 1.35 & 1.34 \\
\hline C-S & $\ldots$. & $\ldots$. & $\ldots$ & $\ldots$. & $\ldots$. & ....... & 1.68 & 1.74 & $\ldots \ldots$ & $\ldots \ldots$ & $\ldots$. & ...... \\
\hline $\mathrm{C}-\mathrm{Cl}$ & $\ldots$. & $\ldots$ & $\ldots$. & $\ldots$. & $\ldots$. & $\ldots$. & ..... & ..... & 1.70 & 1.68 & $\ldots$ & ...... \\
\hline
\end{tabular}

Table 3. Selected bond distances ( $\AA$ ) bond lengths for fluoxetine and its five derivatives.

\begin{tabular}{|c|c|c|c|c|c|c|c|c|c|c|c|c|}
\hline \multirow[b]{2}{*}{ Bond } & \multicolumn{12}{|c|}{ Bond Length (G09) } \\
\hline & \multicolumn{2}{|c|}{ Fluoxetine } & \multicolumn{2}{|c|}{ Patent 1} & \multicolumn{2}{|c|}{ Patent 2} & \multicolumn{2}{|c|}{ Patent 3} & \multicolumn{2}{|c|}{ Patent 4} & \multicolumn{2}{|c|}{ Patent 5} \\
\hline $\mathrm{C}_{1}-\mathrm{N}$ & 1.45 & 1.47 & 1.45 & 1.47 & 1.45 & 1.46 & 1.45 & 1.47 & 1.46 & 1.47 & 1.46 & 1.46 \\
\hline $\mathrm{N}-\mathrm{C}_{7}$ & 1.45 & 1.46 & $\ldots .$. & $\ldots \ldots$ & 1.46 & 1.47 & 1.46 & 1.47 & $\cdots \cdots$ & $\ldots \ldots$ & 1.46 & 1.47 \\
\hline $\mathrm{C}_{12}-\mathrm{O}_{14}$ & 1.45 & 1.48 & 1.45 & 1.45 & 1.48 & 1.46 & 1.46 & 1.50 & $\cdots \cdots$ & $\ldots \ldots$ & 1.46 & 1.48 \\
\hline $\mathrm{O}_{14}-\mathrm{C}_{26}$ & 1.36 & 1.38 & 1.39 & 1.39 & 1.39 & 1.38 & 1.39 & 1.40 & $\cdots \cdots$ & $\ldots \ldots$ & 1.38 & 1.38 \\
\hline $\mathrm{C}_{10}-\mathrm{C}_{12}$ & 1.52 & 1.53 & 1.50 & 1.51 & 1.53 & 1.53 & 1.53 & 1.53 & 1.53 & 1.53 & 1.52 & 1.53 \\
\hline $\mathrm{C}_{7}-\mathrm{C}_{10}$ & 1.52 & 1.53 & 1.52 & 1.48 & 1.53 & 1.53 & 1.53 & 1.53 & 1.54 & 1.55 & 1.53 & 1.53 \\
\hline $\mathrm{C}-\mathrm{S}$ & $\ldots \ldots$ & $\ldots \ldots$ & $\ldots \ldots$ & $\cdots \cdots$ & $\cdots \cdots$ & $\cdots$ & 1.80 & 1.82 & $\ldots \ldots$ & $\ldots \ldots$ & $\ldots \ldots$ & $\cdots \cdot$ \\
\hline $\mathrm{C}-\mathrm{Cl}$ & $\ldots \ldots$ & $\ldots \ldots$ & $\ldots \ldots$ & $\ldots .$. & 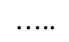 & $\ldots$. & $\ldots \ldots$ & $\ldots \ldots$ & 1.82 & 1.84 & $\ldots \ldots$ & $\ldots \ldots$ \\
\hline
\end{tabular}

Table 4. Selected bond distances $(\AA)$ bond lengths for fluoxetine and its five derivatives.

\begin{tabular}{|c|c|c|c|c|c|c|c|c|c|c|c|c|}
\hline \multirow{3}{*}{ Bond } & \multicolumn{12}{|c|}{ Bond Angles $\left({ }^{\circ}\right)(\mathrm{G} 09)$} \\
\hline & \multicolumn{2}{|c|}{ Fluoxetine } & \multicolumn{2}{|c|}{ Patent 1} & \multicolumn{2}{|c|}{ Patent 2} & \multicolumn{2}{|c|}{ Patent 3} & \multicolumn{2}{|c|}{ Patent 4} & \multicolumn{2}{|c|}{ Patent 5} \\
\hline & AM1 & PM3 & AM1 & PM3 & AM1 & PM3 & AM1 & PM3 & AM1 & PM3 & AM1 & PM3 \\
\hline $\mathrm{C}_{7}-\mathrm{N}_{5}-\mathrm{C}_{10}$ & 112.9 & 110.6 & 109.4 & 110.6 & 113.6 & 110.7 & 113.5 & 110.5 & 113.4 & 110.8 & 113.7 & 110.6 \\
\hline $\mathrm{C}_{7}-\mathrm{C}_{10}-\mathrm{C}_{12}$ & 109.5 & 110.4 & 120.4 & 117.3 & 109.9 & 110.7 & 110.2 & 111.0 & 110.2 & 111.0 & 1109.5 & 110.4 \\
\hline $\mathrm{C}_{12}-\mathrm{O}_{14}-\mathrm{C}_{26}$ & 117.3 & 117.6 & 115.6 & 1.45 & 113.9 & 113.5 & 114.2 & 114.6 & $\cdots \cdots$ & $\cdots \cdots$ & 115.5 & 116.8 \\
\hline $\mathrm{C}_{33}-\mathrm{C}_{36}-\mathrm{F}_{37}$ & 114.6 & 114.1 & 114.8 & 114.9 & $\cdots \cdots$ & $\cdots \cdots$ & $\cdots \cdots$ & $\cdots \cdots$ & $\cdots \cdots$ & $\cdots \cdots$ & $\cdots \cdots$ & ...... \\
\hline $\mathrm{C}_{29}-\mathrm{C}_{33}-\mathrm{C}_{31}$ & 120.5 & 120.6 & 119.5 & 121.0 & 120.9 & 120.2 & 120.4 & 120.0 & $\cdots \cdots$ & $\cdots \cdots$ & $\cdots \cdots$ & ..... \\
\hline
\end{tabular}


Table 5. Selected bond distances $(\AA)$ bond lengths for fluoxetine and its five derivatives.

\begin{tabular}{|c|c|c|c|c|c|c|c|c|c|c|c|c|}
\hline \multirow{3}{*}{ Bond } & \multicolumn{12}{|c|}{ Bond Angles $\left({ }^{\circ}\right)(\mathrm{G} 09)$} \\
\hline & \multicolumn{2}{|c|}{ Fluoxetine } & \multicolumn{2}{|c|}{ Patent 1} & \multicolumn{2}{|c|}{ Patents 2} & \multicolumn{2}{|c|}{ Patent 3} & \multicolumn{2}{|c|}{ Patent 4} & \multicolumn{2}{|c|}{ Patent 5} \\
\hline & $\mathrm{HF}$ & DFT & $\mathrm{HF}$ & DFT & $\mathrm{HF}$ & DFT & $\mathrm{HF}$ & DFT & $\mathrm{HF}$ & DFT & $\mathrm{HF}$ & DFT \\
\hline $\mathrm{C}_{7}-\mathrm{N}_{5}-\mathrm{C}_{10}$ & 110.4 & 110.4 & 110.5 & 110.6 & 111.6 & 111.7 & 111.4 & 111.5 & 110.1 & 110.3 & 112.1 & 112.6 \\
\hline $\mathrm{C}_{7}-\mathrm{C}_{10}-\mathrm{C}_{12}$ & 113.7 & 113.3 & 112.6 & 112.7 & 113.4 & 113.0 & 113.6 & 113.6 & 113.1 & 113.0 & 113.0 & 112.8 \\
\hline $\mathrm{C}_{12}-\mathrm{O}_{14}-\mathrm{C}_{26}$ & 124.2 & 121.2 & 117.6 & 118.9 & 120.1 & 120.8 & 119.9 & 117.9 & $\cdots \cdots$ & $\ldots \ldots$ & 120.7 & 120.3 \\
\hline $\mathrm{C}_{33}-\mathrm{C}_{36}-\mathrm{F}_{37}$ & 113.3 & 113.3 & 112.9 & 113.0 & $\cdots \cdots$ & $\cdots \cdots$ & $\cdots \cdots$ & $\ldots \ldots$ & $\cdots \cdots$ & $\ldots \ldots$ & $\ldots \ldots$ & $\cdots \cdots$ \\
\hline $\mathrm{C}_{29}-\mathrm{C}_{33}-\mathrm{C}_{31}$ & 119.8 & 119.3 & 119.7 & 120.1 & 120.9 & 121.0 & 120.9 & 120.9 & $\ldots$. & $\ldots \ldots$ & $\ldots \ldots$ & -... \\
\hline
\end{tabular}

moments have been calculated in solvent media with (AM1, PM3) and (HF, DFT/B3LYP/6-31G) level for fluoxetine drug and its derivatives. Table 6 and Table 7 list the calculated values of some thermodynamic parameters (such as zero-point vibrational energy, enthalpy, $\mathrm{E}_{\mathrm{HO} о}, \mathrm{E}_{\mathrm{LUMO}}$, Gibbs free energy), $\mathrm{E}_{\mathrm{Hомо}}$, $\mathrm{E}_{\mathrm{LUMO}}$, thermal corrections to (energy, enthalpy, entropy, and Gibbs free energy) fluoxetine and its derivatives. Were obtained using (AM1, PM3) methods, showed that the Patent 3, 4 are more stable than the other patents. The prediction of accurate dipole moments is a very important issue because the magnitude of the dipole moment is strongly related to structure activity of drug. The results obtained using (HF, DFT) method predicts the same evaluation. The value of the dipole moment (D.M) for drugs was also calculated in Table 6 and Table 7. The dipole moment is a measure of the molecular charge distribution. Adirection of the (D.M) in a molecule depends on the centers of positive and negative charges. As a result of calculations, the highest dipole moment was observed for a drug in HF/6-31G (6.121) whereas the smallest one was observed for a drug in PM3 (4.029) the value of dipole moment due to their effect on the charge density of the molecule. The value of the (D.M) for the compounds is a character for the polarity of the compounds mostly, the higher the compound polarity the higher that activity of it. As Table 6, Table 7 shows that Patent 1, 5 is a D.M have a similar D.M to the drug, relatively has the same activity. These parameters may also play an important role in the biological activity of drugs. The vibration entropy and $\mathrm{C}_{\mathrm{V}}$ are found considerably change by changing the methods. The DFT/B3LYP/6-31G result has been given the biggest value for fluoxetine for vibrational entropy (84.47) (Cal/mole-Kelvin) and the biggest vibrational $\mathrm{C}_{\mathrm{v}}$ (73.474) (Cal/mole-Kelvin) value whereas the five derivatives have been given the more stability for Patents 3 and 4. Mostly, DFT method is a more professional way to evaluate the methods of characters due to its modern and complex calculations, there its results more reliable than other method. DFT method gives relatively similar results for the energy evaluations but not for the HOMO, LUMO energies and the dipole moment which give relativity different results as Table 6 and Table 7 show. Because the study is a correlation study, there the difference in results will not affect the study. 
Q. M. A. H. AL-Makhzumi et al.

Table 6. Selected thermodynamic parameters for AM1, PM3 of the drug and its derivativesin G09.

\begin{tabular}{|c|c|c|c|c|c|c|c|c|c|c|c|c|}
\hline \multirow[t]{2}{*}{ Thermodynamic Parameter } & \multicolumn{2}{|c|}{ Fluoxetine } & \multicolumn{2}{|c|}{ Patent 1} & \multicolumn{2}{|c|}{ Patent 2} & \multicolumn{2}{|c|}{ Patent 3} & \multicolumn{2}{|c|}{ Patent 4} & \multicolumn{2}{|c|}{ Patent 5} \\
\hline & AM1 & PM3 & AM1 & PM3 & AM1 & PM3 & AM1 & PM3 & AM1 & PM3 & AM1 & PM3 \\
\hline 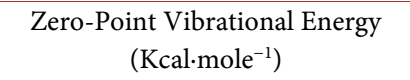 & 205.91 & 199.49 & 186.97 & 182.83 & 240.85 & 232.46 & 219.26 & 211.55 & 170.11 & 164.02 & 214.63 & 207.43 \\
\hline 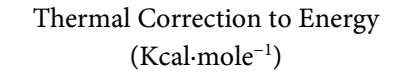 & 219.57 & 213.34 & 196.49 & 195.81 & 254.82 & 246.67 & 233.07 & 225.64 & 181.26 & 175.48 & 227.26 & 220.37 \\
\hline 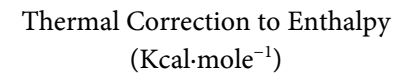 & 220.19 & 213.96 & 197.11 & 196.43 & 255.43 & 247.28 & 233.68 & 226.25 & 181.88 & 176.09 & 227.87 & 220.99 \\
\hline 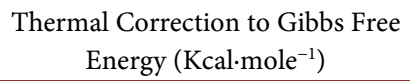 & 170.15 & 163.32 & 159.97 & 146.79 & 203.89 & 195.88 & 182.39 & 175.03 & 138.56 & 132.18 & 181.05 & 226.01 \\
\hline \multicolumn{13}{|l|}{$\mathrm{C}_{\mathrm{V}}(\mathrm{Cal} / \mathrm{mole}-\mathrm{Kelvin})$} \\
\hline Total & 77.14 & 79.43 & 62.09 & 74.45 & 78.52 & 82.06 & 76.38 & 79.93 & 63.40 & 66.06 & 72.33 & 75.52 \\
\hline Translation & 2.981 & 2.981 & 2.981 & 2.981 & 2.981 & 2.981 & 2.981 & 2.981 & 2.981 & 2.981 & 2.981 & 2.981 \\
\hline Rotational & 2.981 & 2.981 & 2.981 & 2.981 & 2.981 & 2.981 & 2.981 & 2.981 & 2.981 & 2.981 & 2.981 & 2.981 \\
\hline Vibrational & 71.15 & 73.474 & 56.14 & 68.49 & 72.55 & 76.10 & 70.42 & $73 . .96$ & 57.45 & 60.10 & 66.37 & 69.55 \\
\hline \multicolumn{13}{|l|}{ S (Entropy) (Cal/mole-Kelvin) } \\
\hline Total & 161.42 & 163.34 & 119.79 & 160.11 & 166.27 & 165.79 & 165.45 & 165.28 & 139.72 & 141.66 & 151.01 & 153.48 \\
\hline Translation & 43.28 & 43.27 & 43.14 & 43.14 & 43.04 & 43.03 & 43.09 & 43.09 & 42.97 & 42.97 & 42.91 & 42.90 \\
\hline Rotational & 35.43 & 35.59 & 35.06 & 35.37 & 35.21 & 35.18 & 35.19 & 35.18 & 34.75 & 34.76 & 34.39 & 34.51 \\
\hline Vibrational & 82.72 & 84.47 & 41.59 & 81.59 & 88.03 & 87.58 & 87.16 & 87.01 & 62.005 & 63.94 & 73.70 & 76.06 \\
\hline $\mathrm{E}_{\text {Homo }}(\mathrm{eV})$ & -9.653 & -9.5 & -9.635 & -9.290 & -9.375 & -9.495 & -9.358 & -9.511 & -9.584 & -9.457 & -9.518 & -9.380 \\
\hline $\mathrm{E}_{\text {Lumo }}(\mathrm{eV})$ & -0.329 & -0.448 & -0.447 & -0.645 & -0.042 & -0.143 & -0.265 & -0.587 & -0.191 & -0.261 & -0.118 & -0.128 \\
\hline $\mathrm{E}_{\mathrm{g}}=\mathrm{E}_{\text {Lumo }}-\mathrm{E}_{\text {Homo }}(\mathrm{eV})$ & 9.323 & 9.065 & 9.188 & 8.644 & 9.334 & 9.352 & 9.093 & 8.924 & 9.3926 & 9.196 & 9.399 & 9.252 \\
\hline Ionization Potential $\left(\mathrm{IE}=-\mathrm{E}_{\text {номо }}\right)$ & 0.329 & 0.448 & 9.635 & 9.290 & 9.375 & 9.495 & 9.358 & 9.511 & 9.584 & 9.457 & 9.518 & 9.380 \\
\hline Electron Affinity $\left(\mathrm{EA}=-\mathrm{E}_{\mathrm{LUMO}}\right)$ & 9.653 & 9.513 & 0.447 & 0.645 & 0.042 & 0.143 & 0.265 & 0.587 & 0.192 & 0.261 & 0.118 & 0.128 \\
\hline Dipole Moment (Debye) & 4.416 & 4.029 & 3.811 & 2.087 & 2.655 & 2.400 & 2.511 & 2.019 & 1.434 & 1.685 & 4.287 & 4.506 \\
\hline
\end{tabular}

\subsection{Theoretical Atomic Charge Calculation}

Mulliken atomic charge calculation plays an important role in the application of quantum chemical calculation to molecule system [12]. Since the charge distribution on the molecule has an important for the vibration spectra.

Calculated atomic charges are a different matter. Atomic charges can be defined in various ways. Mulliken atomic charges are commonly used in molecular orbital theory and are used in and G09. They have varying values according to the basis set and the method of calculation [13]. Table 8 and Table 9 show effective atomic charge calculations which have an important role in the application of quantum chemical calculation to the molecular system the atomic charge levels to the dipole moment, molecular polarization, electronic structure of drug, and the comparison of the different methods to describe the electron distribution of the drugs with its derivatives. Milliken charge distributions were calculated by determining the electron population of each atom as defined by the four 
Table 7. Selected thermodynamic parameters for HF, DFT of the drug and its derivatives in G09.

\begin{tabular}{|c|c|c|c|c|c|c|c|c|c|c|c|c|}
\hline \multirow[t]{2}{*}{ Thermodynamic Parameter } & \multicolumn{2}{|c|}{ Fluoxetine } & \multicolumn{2}{|c|}{ Patent 1} & \multicolumn{2}{|c|}{ Patent 2} & \multicolumn{2}{|c|}{ Patent 3} & \multicolumn{2}{|c|}{ Patent 4} & \multicolumn{2}{|c|}{ Patent 5} \\
\hline & $\mathrm{HF}$ & DFT & $\mathrm{HF}$ & DFT & $\mathrm{HF}$ & DFT & $\mathrm{HF}$ & DFT & $\mathrm{HF}$ & DFT & $\mathrm{HF}$ & DFT \\
\hline $\begin{array}{l}\text { Zero-Point Vibrational Energy } \\
\qquad\left(\mathrm{Kcal} \mathrm{mole}^{-1}\right)\end{array}$ & 217.78 & 202.77 & 198.47 & 184.63 & 255.59 & 238.74 & 232.84 & 216.89 & 180.01 & 167.59 & 227.68 & 212.55 \\
\hline $\begin{array}{l}\text { Thermal Correction to Energy } \\
\left(\mathrm{Kcal} / \mathrm{mole}^{-1}\right)\end{array}$ & 230.67 & 216.51 & 209.90 & 197.70 & 268.83 & 252.73 & 245.99 & 230.83 & 190.63 & 178.97 & 239.61 & 225.26 \\
\hline $\begin{array}{l}\text { Thermal Correction to Enthalpy } \\
\qquad\left(\mathrm{Kcal} / \mathrm{mole}^{-1}\right)\end{array}$ & 231.28 & 217.12 & 210.49 & 198.32 & 269.44 & 253.34 & 246.60 & 231.45 & 191.25 & 179.58 & 240.22 & 225.87 \\
\hline $\begin{array}{c}\text { Thermal Correction to Gibbs Free } \\
\text { Energy }\left(\mathrm{Kcal} / \mathrm{mole}^{-1}\right)\end{array}$ & 183.28 & 167.58 & 165.69 & 212.17 & 220.39 & 203.03 & 197.53 & 180.83 & 149.29 & 135.76 & 195.03 & 179.08 \\
\hline \multicolumn{13}{|l|}{$\mathrm{C}_{\mathrm{V}}(\mathrm{Cal} /$ mole-Kelvin $)$} \\
\hline Total & 73.11 & 79.23 & 66.66 & 74.98 & 74.63 & 80.67 & 73.14 & 79.02 & 60.54 & 65.63 & 68.43 & 74.20 \\
\hline Translation & 2.981 & 2.981 & 2.981 & 2.981 & 2.981 & 2.981 & 2.981 & 2.981 & 2.981 & 2.981 & 2.981 & 2.981 \\
\hline Rotational & 2.981 & 2.981 & 2.981 & 2.981 & 2.981 & 2.981 & 2.981 & 2.981 & 2.981 & 2.981 & 2.981 & 2.981 \\
\hline Vibrational & 69.14 & 73.27 & 60.70 & 69.03 & 68.66 & 74.70 & 67.17 & 73.05 & 54.58 & 59.67 & 62.47 & 68.24 \\
\hline \multicolumn{13}{|l|}{ S (Entropy) (Cal/mole-Kelvin) } \\
\hline Total & 154.83 & 159.79 & 150.27 & 157.75 & 158.20 & 162.31 & 158.28 & 163.30 & 135.32 & 141.36 & 145.76 & 150.49 \\
\hline Translation & 43.28 & 43.27 & 42.95 & 43.14 & 43.03 & 43.03 & 43.09 & 43.09 & 42.97 & 42.97 & 42.90 & 42.90 \\
\hline Rotational & 35.40 & 35.44 & 35.01 & 35.16 & 35.29 & 35.35 & 35.31 & 35.34 & 34.82 & 34.85 & 34.37 & 34.55 \\
\hline Vibrational & 76.15 & 81.07 & 72.32 & 79.45 & 79.87 & 83.93 & 79.88 & 84.34 & 57.53 & 63.54 & 68.49 & 73.48 \\
\hline $\mathrm{E}_{\text {Homo }}(\mathrm{eV})$ & -9.215 & -5.754 & -8.822 & -5.945 & -8.716 & -5.699 & -8.790 & -5.723 & -9.061 & -5.989 & -9.176 & -5.582 \\
\hline $\mathrm{E}_{\text {Lumo }}(\mathrm{eV})$ & 3.107 & -0.777 & 2.598 & -1.024 & 3.573 & -0.295 & 3.175 & -0.859 & 3.110 & -0.643 & 3.326 & -0.402 \\
\hline $\mathrm{E}_{\mathrm{g}}=\mathrm{E}_{\text {Lumo }}-\mathrm{E}_{\text {Homo }}(\mathrm{eV})$ & 12.321 & 4.977 & 11.419 & 4.920 & 12.289 & 5.403 & 11.965 & 4.864 & 12.171 & 5.3466 & 12.502 & 5.1804 \\
\hline Ionization Potential $\left(\mathrm{E}=-\mathrm{E}_{\text {номо }}\right)$ & -3.107 & 0.777 & 8.822 & 5.945 & 8.716 & 5.699 & 8.790 & 5.723 & 9.061 & 5.989 & 9.176 & 5.582 \\
\hline Electron Affinity $\left(\mathrm{EA}=-\mathrm{E}_{\mathrm{LUMO}}\right)$ & 9.215 & 5.754 & -2.598 & 1.024 & -3.573 & 0.295 & -3.175 & 0.859 & -3.110 & 0.643 & -3.326 & 0.402 \\
\hline Dipole Moment (Debye) & 6.121 & 5.869 & 5.880 & 6.320 & 3.394 & 3.094 & 3.047 & 2.983 & 4.438 & 4.152 & 5.445 & 4.787 \\
\hline
\end{tabular}

methods. The results in the (AM1, PM3, HF, and DFT) were in Table 8 and Table 9. The charge change with the method, basis set presumably occurs due to polarization. In the atomic charge calculation $\mathrm{O}_{16}, \mathrm{~N}_{5}$ atoms exhibit a substantial negative charge, which is donor atom. But $\mathrm{S}$ and $\mathrm{C}_{8}, \mathrm{C}_{11}, \mathrm{~S}_{35}$ atoms exhibit a positive charge, which is an acceptor atom (See Table 8, Table 9).

\subsection{HOMO and LUMO Molecular Orbital}

In principle, there are several ways to calculate the excitation energies. The simplest one involves the difference between the highest occupied molecular orbital (HOMO) of a neutral system, which is a key parameter in determining molecular properties [13]. The Eigen values of HOMO ( $\pi$ donor) and LUMO ( $\pi$ acceptor) and their energy gap between HOMO and LUMO characterizes the molecular chemical stability. The energy gap reflects the chemical activity of the molecules [13]. The relatively large LUMO-HOMO energy gap of the studied molecule indicates that it can be considered as kinetically stable. In addition, energy 
Q. M. A. H. AL-Makhzumi et al.

Table 8. Selected atomic charges of drugs and its derivatives in AM1, PM3 in Gaussian G09.

\begin{tabular}{|c|c|c|c|c|c|c|c|c|c|c|c|c|}
\hline \multirow[t]{2}{*}{ Atoms } & \multicolumn{2}{|c|}{ Fluoxetine } & \multicolumn{2}{|c|}{ Patent 1} & \multicolumn{2}{|c|}{ Patent 2} & \multicolumn{2}{|c|}{ Patent 3} & \multicolumn{2}{|c|}{ Patent4 } & \multicolumn{2}{|c|}{ Patent 5} \\
\hline & AM1 & PM3 & AM1 & PM3 & AM1 & PM3 & AM1 & PM3 & AM1 & PM3 & AM1 & PM3 \\
\hline $1 \mathrm{C}$ & -0.132 & -0.101 & ........ & ........ & -0.136 & -0.116 & -0.137 & -0.116 & ........ & ........ & -0.129 & -0.093 \\
\hline $2 \mathrm{H}$ & 0.083 & 0.046 & ........ & ....... & 0.086 & 0.053 & 0.086 & 0.045 & ....... & ....... & 0.087 & 0.048 \\
\hline $3 \mathrm{H}$ & 0.056 & 0.032 & $\ldots \ldots$ & $\ldots \ldots$ & 0.053 & 0.032 & 0.052 & 0.032 & $\ldots \ldots$ & $\ldots \ldots$. & 0.058 & 0.035 \\
\hline $4 \mathrm{H}$ & 0.087 & 0.046 & $\ldots \ldots$ & $\ldots \ldots$ & 0.083 & 0.048 & 0.083 & 0.053 & $\ldots \ldots$. & $\ldots \ldots$ & 0.086 & 0.048 \\
\hline $5 \mathrm{~N}$ & -0.323 & -0.087 & -0.370 & -0.065 & -0.323 & -0.085 & -0.323 & -0.084 & -0.365 & -0.065 & -0.278 & -0.096 \\
\hline $6 \mathrm{H}$ & 0.162 & 0.056 & 0.156 & 0.037 & 0.166 & 0.057 & 0.167 & 0.053 & 0.150 & 0.037 & ........ & $\ldots \ldots$ \\
\hline $8 \mathrm{H}$ & $\ldots \ldots$. & $\ldots \ldots$. & 0.150 & 0.034 & $\ldots \ldots$ & $\ldots \ldots$ & $\ldots \ldots$ & $\ldots \ldots$ & 0.157 & 0.034 & ........ & $\ldots \ldots$ \\
\hline $8 \mathrm{C}$ & -0.081 & -0.091 & -0.080 & -0.101 & -0.078 & -0.091 & -0.076 & -0.087 & -0.087 & -0.065 & -0.076 & -0.082 \\
\hline $9 \mathrm{H}$ & 0.093 & 0.060 & 0.089 & 0.056 & 0.065 & 0.048 & 0.094 & 0.062 & 0.090 & 0.104 & 0.095 & 0.049 \\
\hline $10 \mathrm{H}$ & 0.065 & 0.048 & 0.056 & 0.041 & 0.092 & 0.059 & 0.062 & 0.045 & 0.059 & 0.057 & 0.068 & 0.045 \\
\hline $11 \mathrm{C}$ & -0.165 & -0.143 & -0.126 & -0.055 & -0.184 & -0.153 & -0.180 & -0.151 & -0.160 & -0.116 & -0.161 & -0.128 \\
\hline $12 \mathrm{H}$ & 0.104 & 0.076 & 0.097 & 0.061 & 0.098 & 0.070 & 0.099 & 0.077 & 0.098 & 0.067 & 0.103 & 0.077 \\
\hline $13 \mathrm{H}$ & 0.106 & 0.077 & 0.094 & 0.062 & 0.099 & 0.076 & 0.100 & 0.073 & 0.093 & 0.068 & 0.102 & 0.071 \\
\hline $14 \mathrm{C}$ & 0.068 & 0.106 & ........ & $\ldots \ldots$ & 0.068 & 0.119 & 0.102 & 0.152 & -0.035 & -0.011 & 0.070 & 0.111 \\
\hline $15 \mathrm{H}$ & 0.113 & 0.085 & ......... & ........ & 0.107 & 0.072 & 0.109 & 0.079 & 0.116 & 0.084 & 0.108 & 0.082 \\
\hline $16 \mathrm{O}$ & -0.217 & -0.193 & -0.228 & -0.200 & -0.244 & -0.222 & -0.245 & -0.220 & $\ldots$. & $\ldots \ldots$ & -0.231 & -0.192 \\
\hline $17 \mathrm{C}$ & $\ldots \ldots$ & $\ldots$. & 0.005 & 0.083 & $\ldots \ldots$ & ........ & $\ldots \ldots$ & ....... & $\ldots \ldots$ & $\ldots \ldots$ & $\ldots \ldots$ & ........ \\
\hline $18 \mathrm{H}$ & $\ldots \ldots$ & $\ldots \ldots$ & 0.110 & 0.076 & $\ldots \ldots$ & $\ldots \ldots$ & $\ldots$. & $\ldots .$. & $\ldots \ldots$ & $\ldots .$. & $\ldots \ldots$ & $\ldots \ldots$. \\
\hline $19 \mathrm{H}$ & $\ldots \ldots$ & $\ldots$. & 0.107 & 0.071 & $\ldots \ldots$ & $\ldots \ldots \ldots$ & $\ldots$. & $\ldots \ldots$ & $\ldots$. & $\ldots \ldots$ & $\ldots \ldots$ & ........ \\
\hline $20 \mathrm{C}$ & ....... & ........ & ....... & ........ & ........ & $\ldots \ldots$ & $\ldots \ldots$ & $\ldots \ldots$ & $\ldots \ldots$ & $\ldots \ldots$ & -0.134 & -0.104 \\
\hline $21 \mathrm{H}$ & $\ldots \ldots$ & $\ldots \ldots$ & ....... & $\ldots \ldots$ & ........ & $\ldots \ldots$ & $\ldots \ldots$ & $\ldots \ldots$ & ........ & $\ldots \ldots$ & 0.087 & 0.049 \\
\hline $22 \mathrm{H}$ & ....... & ....... & ....... & ....... & ........ & $\ldots \ldots$ & ....... & $\ldots \ldots$ & ........ & $\ldots \ldots$ & 0.057 & 0.036 \\
\hline $23 \mathrm{H}$ & ....... & ....... & $\ldots \ldots$ & $\ldots \ldots$ & ........ & ........ & ....... & $\ldots \ldots$ & ........ & ....... & 0.087 & 0.059 \\
\hline $24 \mathrm{C}$ & -0.127 & -0.117 & -0.056 & -0.092 & -0.095 & -0.110 & -0.412 & -0.306 & -0.062 & -0.065 & -0.122 & -0.133 \\
\hline $25 \mathrm{C}$ & -0.123 & -0.085 & -0.124 & -0.083 & -0.126 & -0.086 & -0.140 & -0.093 & -0.125 & -0.101 & -0.116 & -0.089 \\
\hline $26 \mathrm{C}$ & -0.113 & -0.092 & -0.117 & -0.091 & -0.115 & -0.094 & -0.175 & -0.125 & -0.127 & -0.106 & -0.124 & -0.084 \\
\hline $27 \mathrm{C}$ & -0.137 & -0.111 & -0.082 & -0.051 & -0.139 & -0.111 & $\ldots \ldots$ & ....... & -0.121 & -0.108 & -0.148 & -0.110 \\
\hline $28 \mathrm{C}$ & -0.139 & -0.108 & -0.083 & -0.044 & -0.141 & -0.112 & $\ldots \ldots$ & $\ldots \ldots$ & -0.129 & -0.144 & -0.139 & -0.111 \\
\hline $29 \mathrm{C}$ & -0.126 & -0.095 & -0.171 & -0.187 & -0.130 & -0.100 & -0.440 & -0.285 & -0.085 & -0.087 & -0.131 & -0.098 \\
\hline $30 \mathrm{H}$ & 0.146 & 0.115 & 0.161 & 0.125 & 0.145 & 0.116 & 0.163 & 0.137 & 0.151 & 0.127 & 0.149 & 0.116 \\
\hline $31 \mathrm{H}$ & 0.146 & 0.121 & 0.159 & 0.125 & 0.147 & 0.117 & 0.162 & 0.135 & 0.155 & 0.121 & 0.144 & 0.117 \\
\hline $32 \mathrm{H}$ & 0.145 & 0.113 & 0.160 & 0.125 & 0.145 & 0.113 & 0.186 & 0.161 & 0.152 & 0.116 & 0.143 & 0.114 \\
\hline $33 \mathrm{H}$ & 0.144 & 0.113 & 0.160 & 0.127 & 0.144 & 0.113 & $\ldots \ldots$ & $\ldots \ldots$ & 0.154 & 0.123 & 0.144 & 0.113 \\
\hline $34 \mathrm{H}$ & 0.143 & 0.112 & $\ldots \ldots$ & $\ldots$. & 0.142 & 0.112 & $\ldots \ldots$ & $\ldots \ldots$ & $\ldots \ldots$ & $\ldots \ldots$ & 0.143 & 0.112 \\
\hline $35 \mathrm{~S}$ & $\ldots \ldots$ & $\ldots \ldots$ & $\ldots \ldots$ & $\ldots$. & $\ldots \ldots$ & $\ldots \ldots$ & 0.591 & 0.280 & ........ & $\ldots \ldots$ & $\ldots \ldots$ & $\ldots \ldots$ \\
\hline $36 \mathrm{C}$ & 0.114 & 0.130 & 0.066 & 0.065 & 0.037 & 0.045 & 0.038 & 0.043 & -0.069 & -0.078 & 0.021 & 0.062 \\
\hline $37 \mathrm{C}$ & -0.172 & -0.158 & -0.168 & -0.148 & -0.160 & -0.131 & -0.146 & -0.121 & -0.120 & -0.097 & -0.148 & -0.159 \\
\hline $38 \mathrm{C}$ & -0.208 & -0.195 & -0.200 & -0.179 & -0.146 & -0.123 & -0.164 & -0.131 & -0.120 & -0.090 & 0.056 & 0.006 \\
\hline
\end{tabular}




\begin{tabular}{|c|c|c|c|c|c|c|c|c|c|c|c|c|}
\hline \multicolumn{13}{|c|}{ Continued } \\
\hline $39 \mathrm{C}$ & -0.042 & -0.011 & -0.099 & -0.070 & -0.085 & -0.085 & -0.089 & -0.091 & -0.127 & -0.104 & -0.122 & -0.100 \\
\hline $40 \mathrm{C}$ & -0.051 & 0.008 & -0.101 & -0.064 & -0.116 & -0.091 & -0.116 & -0.086 & -0.127 & -0.149 & -0.151 & -0.114 \\
\hline $41 \mathrm{C}$ & -0.217 & -0.244 & -0.111 & -0.116 & -0.116 & -0.085 & -0.113 & -0.084 & -0.088 & -0.100 & -0.124 & -0.078 \\
\hline $42 \mathrm{H}$ & 0.165 & 0.134 & 0.153 & 0.125 & 0.153 & 0.123 & 0.151 & 0.124 & 0.152 & 0.127 & 0.157 & 0.134 \\
\hline $43 \mathrm{H}$ & 0.164 & 0.140 & 0.152 & 0.131 & 0.153 & 0.123 & 0.152 & 0.124 & 0.153 & 0.119 & 0.153 & 0.116 \\
\hline $44 \mathrm{H}$ & 0.160 & 0.121 & 0.145 & 0.115 & 0.147 & 0.117 & 0.147 & 0.117 & 0.156 & 0.124 & 0.160 & 0.119 \\
\hline $45 \mathrm{H}$ & 0.161 & 0.124 & 0.146 & 0.116 & 0.147 & 0.117 & 0.147 & 0.117 & 0.156 & 0.124 & 0.154 & 0.128 \\
\hline $46 \mathrm{C}$ & 0.474 & 0.411 & 0.469 & 0.469 & -0.164 & -0.096 & -0.133 & -0.076 & $\ldots$. & $\cdots \cdots$ & $\ldots$. & $\ldots$. \\
\hline $47 \mathrm{~F}$ & -0.177 & -0.150 & -0.174 & -0.147 & $\ldots$. & $\ldots$. & $\ldots$. & $\ldots$. & $\ldots$. & ..... & $\cdots \cdots$ & $\cdots \cdots$ \\
\hline $48 \mathrm{~F}$ & -0.175 & -0.141 & -0.173 & -0.145 & 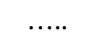 & ..... & $\ldots \ldots$ & $\ldots \ldots$ & ..... & ..... & $\cdots \cdots$ & $\cdots \cdots$ \\
\hline $49 \mathrm{~F}$ & -0.175 & -0.149 & -0.172 & -0.142 & $\ldots$. & $\ldots$. & $\ldots .$. & $\ldots .$. & $\ldots$. & $\ldots$. & -0.108 & -0.097 \\
\hline $50 \mathrm{H}$ & $\ldots \ldots$ & $\ldots \ldots$ & $\ldots \ldots$ & ........ & 0.100 & 0.066 & 0.103 & 0.067 & $\ldots$. & $\ldots$. & $\ldots$. & ..... \\
\hline $51 \mathrm{H}$ & ........ & $\ldots \ldots$ & $\ldots \ldots$ & ......... & 0.103 & 0.067 & 0.075 & 0.066 & $\ldots$. & $\ldots$. & $\ldots$. & ..... \\
\hline $52 \mathrm{H}$ & ........ & ........ & ....... & ......... & ..... & $\ldots \ldots$ & $\ldots \ldots$ & $\ldots \ldots$ & $\ldots$. & $\ldots \ldots$ & $\ldots .$. & $\cdots \cdots$ \\
\hline $53 \mathrm{C}$ & $\ldots \ldots$ & $\ldots \ldots$ & $\ldots \ldots$ & ......... & -0.019 & 0.071 & -0.017 & 0.076 & $\ldots .$. & $\ldots \ldots$ & ..... & ..... \\
\hline $54 \mathrm{H}$ & $\ldots \ldots$ & $\ldots \ldots$ & $\ldots \ldots$ & $\ldots \ldots$ & 0.106 & 0.054 & 0.075 & 0.034 & $\ldots$. & $\ldots \ldots$ & $\ldots .$. & $\ldots .$. \\
\hline $55 \mathrm{H}$ & $\ldots \ldots$ & $\ldots \ldots$ & ....... & $\ldots \ldots$ & 0.076 & 0.037 & 0.075 & 0.034 & $\ldots$. & $\ldots$. & $\ldots$. & ..... \\
\hline $56 \mathrm{O}$ & $\ldots \ldots$ & $\ldots \ldots$ & $\ldots \ldots$ & $\ldots \ldots$ & 0.385 & -0.341 & -0.361 & -0.344 & $\ldots$. & $\ldots \ldots$ & $\ldots .$. & ..... \\
\hline $57 \mathrm{H}$ & ....... & $\ldots \ldots$ & $\ldots \ldots$ & $\ldots \ldots$ & 0.216 & 0.203 & 0.219 & 0.207 & ..... & $\ldots \ldots$ & ..... & $\cdots \cdots$ \\
\hline $58 \mathrm{Cl}_{2}$ & $\ldots \ldots$ & ........ & ........ & ........ & $\ldots \ldots$ & ........ & $\ldots \ldots$ & $\ldots \ldots$ & -0.020 & -0.064 & ..... & $\ldots \ldots$ \\
\hline
\end{tabular}

of the HOMO is directly related to the ionization potential, while the energy of the LUMO is directly related to the electron affinity. The energy gaps are largely responsible for the chemical and spectroscopic properties of the molecules [13]. LUMO-HOMO gap energy of fluoxetine and its derivatives are calculated by four methods and various levels which are given in Table 6, Table 7 and Figure 3. As a result, at the HOMO energies calculated by the (AM1, PM3, HF, DFT) methods by using the Gaussian 09 program by the different basis sets with diffuse function are higher than those of the other basis sets. The HOMO energy value for drug calculated at (AM1, PM3, HF, DFT) (-9.6533, -9.5124, -9.2147, $-5.754) \mathrm{eV}$. The biggest HOMO energy value calculated at DFT/6-31G/B3LYP, whereas the smallest one is calculated at AM1. The biggest LUMO energy value is (3.1066) eV obtained using HF/6031G, band energy gap $\left(\mathrm{E}_{\mathrm{g}}\right)$ value is (12.3213) eV obtained using HF/6-31G (Table 6, Table 7 and Figure 3).

\subsection{Ionization Energy}

The ionization energy associated with a relationship with a higher energy of occupied orbital as follows [14] [15]. IE $=-$ EHOMO the high value in the energy of ionization mean the high stability of the molecule and on the other hand, the ionization energy means high effectiveness of the molecule. The calculated ionization energy of the drug fluoxetine and its derivatives have studied theoretically by using G09W program according to the methods (AM1, PM3, HF, and DFT) 
Q. M. A. H. AL-Makhzumi et al.

Table 9. Selected atomic charges of drugs and its derivatives in HF, DFT in Gaussian 09.

\begin{tabular}{|c|c|c|c|c|c|c|c|c|c|c|c|c|}
\hline \multirow[t]{2}{*}{ Atoms } & \multicolumn{2}{|c|}{ Fluoxetine } & \multicolumn{2}{|c|}{ Patent 1} & \multicolumn{2}{|c|}{ Patent 2} & \multicolumn{2}{|c|}{ Patent 3} & \multicolumn{2}{|c|}{ Patent 4} & \multicolumn{2}{|c|}{ Patent 5} \\
\hline & $\mathrm{HF}$ & DFT & $\mathrm{HF}$ & DFT & $\mathrm{HF}$ & DFT & $\mathrm{HF}$ & DFT & $\mathrm{HF}$ & DFT & $\mathrm{HF}$ & DFT \\
\hline $1 \mathrm{C}$ & -0.240 & -0.261 & $\ldots \ldots \ldots$ & $\ldots \ldots$ & -0.244 & -0.267 & -0.244 & -0.268 & $\ldots \ldots$. & $\ldots \ldots$. & 0.243 & -0.257 \\
\hline $2 \mathrm{H}$ & 0.163 & 0.148 & $\ldots \ldots$. & $\ldots \ldots$ & 0.164 & 0.149 & 0.169 & 0.151 & $\ldots \ldots$. & $\ldots \ldots$ & 0.169 & 0.147 \\
\hline $3 \mathrm{H}$ & 0.137 & 0.119 & $\ldots \ldots$ & $\ldots \ldots$ & 0.137 & 0.119 & 0.138 & 0.128 & ....... & $\ldots \ldots$ & 0.169 & 0.153 \\
\hline $4 \mathrm{H}$ & 0.163 & 0.148 & $\ldots \ldots$ & $\ldots \ldots$ & 0.168 & 0.151 & 0.163 & 0.149 & $\ldots \ldots$. & $\ldots \ldots$ & 0.135 & 0.149 \\
\hline $5 \mathrm{~N}$ & -0.787 & -0.596 & -0.889 & -0.733 & -0.780 & -0.590 & -0.781 & -0.589 & -0.888 & -0.736 & -0.693 & -0.455 \\
\hline $6 \mathrm{H}$ & 0.334 & 0.291 & 0.331 & 0.291 & 0.339 & 0.295 & 0.341 & 0.290 & 0.336 & 0.290 & $\ldots \ldots$ & $\ldots$ \\
\hline $8 \mathrm{H}$ & $\ldots \ldots$ & $\ldots \ldots$ & 0.339 & 0.294 & $\ldots \ldots$ & $\ldots \ldots$ & $\ldots \ldots$ & $\ldots$. & 0.331 & 0.294 & ........ & $\ldots$ \\
\hline $8 \mathrm{C}$ & -0.084 & -0.101 & -0.079 & -0.103 & -0.107 & -0.123 & -0.106 & -0.123 & -0.094 & -0.119 & -0.092 & -0.104 \\
\hline $9 \mathrm{H}$ & 0.174 & 0.116 & 0.147 & 0.118 & 0.173 & 0.121 & 0.153 & 0.123 & 0.169 & 0.124 & 0.144 & 0.148 \\
\hline $10 \mathrm{H}$ & 0.144 & 0.148 & 0.170 & 0.143 & 0.150 & 0.144 & 0.177 & 0.140 & 0.152 & 0.144 & 0.170 & 0.114 \\
\hline $11 \mathrm{C}$ & -0.276 & -0.239 & -0.315 & -0.307 & -0.244 & -0.220 & -0.292 & -0.229 & -0.275 & -0.228 & -0.274 & -0.234 \\
\hline $12 \mathrm{H}$ & 0.180 & 0.151 & 0.173 & 0.139 & 0.168 & 0.148 & 0.186 & 0.149 & 0.165 & 0.140 & 0.104 & 0.148 \\
\hline $13 \mathrm{H}$ & 0.180 & 0.152 & 0.177 & 0.140 & 0.137 & 0.146 & 0.176 & 0.150 & 0.179 & 0.144 & 0.177 & 0.155 \\
\hline $14 \mathrm{C}$ & 0.120 & 0.005 & $\ldots \ldots$ & $\ldots \ldots$ & 0.100 & 0.103 & 0.120 & 0.130 & -0.266 & -0.274 & 0.109 & 0.099 \\
\hline $15 \mathrm{H}$ & 0.202 & 0.160 & $\ldots \ldots \ldots$ & $\ldots \ldots$. & 0.194 & 0.156 & 0.200 & 0.161 & 0.211 & 0.156 & 0.199 & 0.159 \\
\hline $16 \mathrm{O}$ & -0.830 & -0.589 & -0.790 & -0.598 & -0.804 & -0.608 & -0.795 & -0.587 & ..... & $\ldots \ldots$ & -0.799 & -0.588 \\
\hline $17 \mathrm{C}$ & $\ldots \ldots$ & $\ldots$. & -0.001 & -0.078 & ........ & ........ & ...... & ....... & $\ldots \ldots$ & $\ldots \ldots$ & $\ldots \ldots$ & ........ \\
\hline $18 \mathrm{H}$ & $\ldots \ldots$ & ...... & 0.194 & 0.182 & ........ & $\ldots \ldots$ & ..... & $\ldots$. & ...... & ..... & $\ldots \ldots$ & ........ \\
\hline $19 \mathrm{H}$ & $\ldots \ldots$ & ..... & 0.194 & 0.180 & ........ & ........ & ..... & $\ldots \ldots$ & $\cdots \cdots$ & ...... & ........ & ........ \\
\hline $20 \mathrm{C}$ & ........ & ........ & ........ & ........ & $\ldots \ldots$ & $\ldots \ldots$ & ........ & ........ & ........ & ........ & -0.230 & -0.257 \\
\hline $21 \mathrm{H}$ & $\ldots \ldots$ & ........ & $\ldots \ldots$ & $\ldots \ldots$ & $\ldots \ldots$ & $\ldots \ldots$ & ........ & $\ldots \ldots$ & ........ & $\ldots \ldots$ & 0.166 & 0.147 \\
\hline $22 \mathrm{H}$ & ........ & ........ & ........ & ........ & $\ldots \ldots$ & $\ldots \ldots$ & $\ldots \ldots$ & $\ldots \ldots$ & $\ldots \ldots$ & $\ldots \ldots$ & 0.168 & 0.149 \\
\hline $23 \mathrm{H}$ & $\ldots \ldots$ & ........ & $\ldots \ldots$ & $\ldots \ldots$ & $\ldots \ldots$ & $\ldots \ldots$ & $\ldots \ldots$ & $\ldots \ldots$ & $\ldots \ldots$ & $\ldots \ldots$ & 0.135 & 0.116 \\
\hline $24 \mathrm{C}$ & -0.127 & -0.117 & 0.009 & 0.059 & -0.101 & -0.106 & -0.552 & -0.451 & -0.100 & -0.103 & -0.045 & -0.089 \\
\hline $25 \mathrm{C}$ & -0.123 & -0.085 & -0.213 & -0.136 & -0.216 & -0.145 & -0.139 & -0.074 & -0.155 & -0.122 & -0.194 & -0.161 \\
\hline $26 \mathrm{C}$ & -0.214 & -0.160 & -0.213 & -0.152 & -0.214 & -0.148 & -0.107 & -0.047 & -0.214 & -0.106 & -0.214 & -0.150 \\
\hline $27 \mathrm{C}$ & -0.215 & -0.144 & -0.148 & -0.123 & -0.224 & -0.145 & ...... & ....... & -0.210 & -0.154 & 0.219 & -0.145 \\
\hline $28 \mathrm{C}$ & -0.215 & -0.143 & -0.148 & -0.118 & -0.218 & -0.164 & ....... & $\ldots \ldots$ & -0.162 & -0.146 & -0.213 & -0.143 \\
\hline $29 \mathrm{C}$ & -0.214 & -0.160 & -0.206 & -0.017 & -0.195 & -0.133 & -0.391 & -0.299 & -0.312 & -0.240 & -0.216 & -0.133 \\
\hline $30 \mathrm{H}$ & 0.212 & 0.147 & 0.246 & 0.164 & 0.229 & 0.150 & 0.245 & 0.192 & 0.255 & 0.173 & 0.235 & 0.151 \\
\hline $31 \mathrm{H}$ & 0.223 & 0.147 & 0.246 & 0.171 & 0.218 & 0.146 & 0.240 & 0.165 & 0.253 & 0.157 & 0.228 & 0.147 \\
\hline $32 \mathrm{H}$ & 0.223 & 0.148 & 0.266 & 0.175 & 0.219 & 0.145 & 0.253 & 0.169 & 0.236 & 0.160 & 0.221 & 0.146 \\
\hline $33 \mathrm{H}$ & 0.230 & 0.153 & 0.266 & 0.174 & 0.220 & 0.145 & $\ldots \ldots$ & $\ldots \ldots$ & 0.238 & 0.173 & 0.221 & 0.147 \\
\hline $34 \mathrm{H}$ & 0.233 & 0.152 & $\ldots$. & $\ldots$. & 0.219 & 0.145 & $\ldots \ldots$ & $\ldots \ldots$ & ...... & $\ldots \ldots$ & 0.222 & 0.152 \\
\hline $35 \mathrm{~S}$ & $\ldots$ & ...... & $\ldots \ldots$ & ..... & ...... & $\ldots \ldots$ & 0.453 & 0.380 & $\ldots \ldots$ & ...... & ...... & $\ldots \ldots$ \\
\hline $36 \mathrm{C}$ & 0.440 & 0.311 & 0.336 & 0.285 & 0.353 & 0.283 & 0.340 & 0.266 & -0.127 & -0.133 & 0.355 & 0.279 \\
\hline $37 \mathrm{C}$ & -0.235 & -0.138 & -0.205 & -0.148 & -0.216 & -0.152 & -0.210 & -0.134 & -0.155 & -0.160 & -0.203 & -0.133 \\
\hline $38 \mathrm{C}$ & -0.250 & -0.155 & -0.206 & -0.144 & -0.210 & -0.141 & -0.219 & -0.138 & -0.151 & -0.107 & -0.359 & -0.149 \\
\hline
\end{tabular}




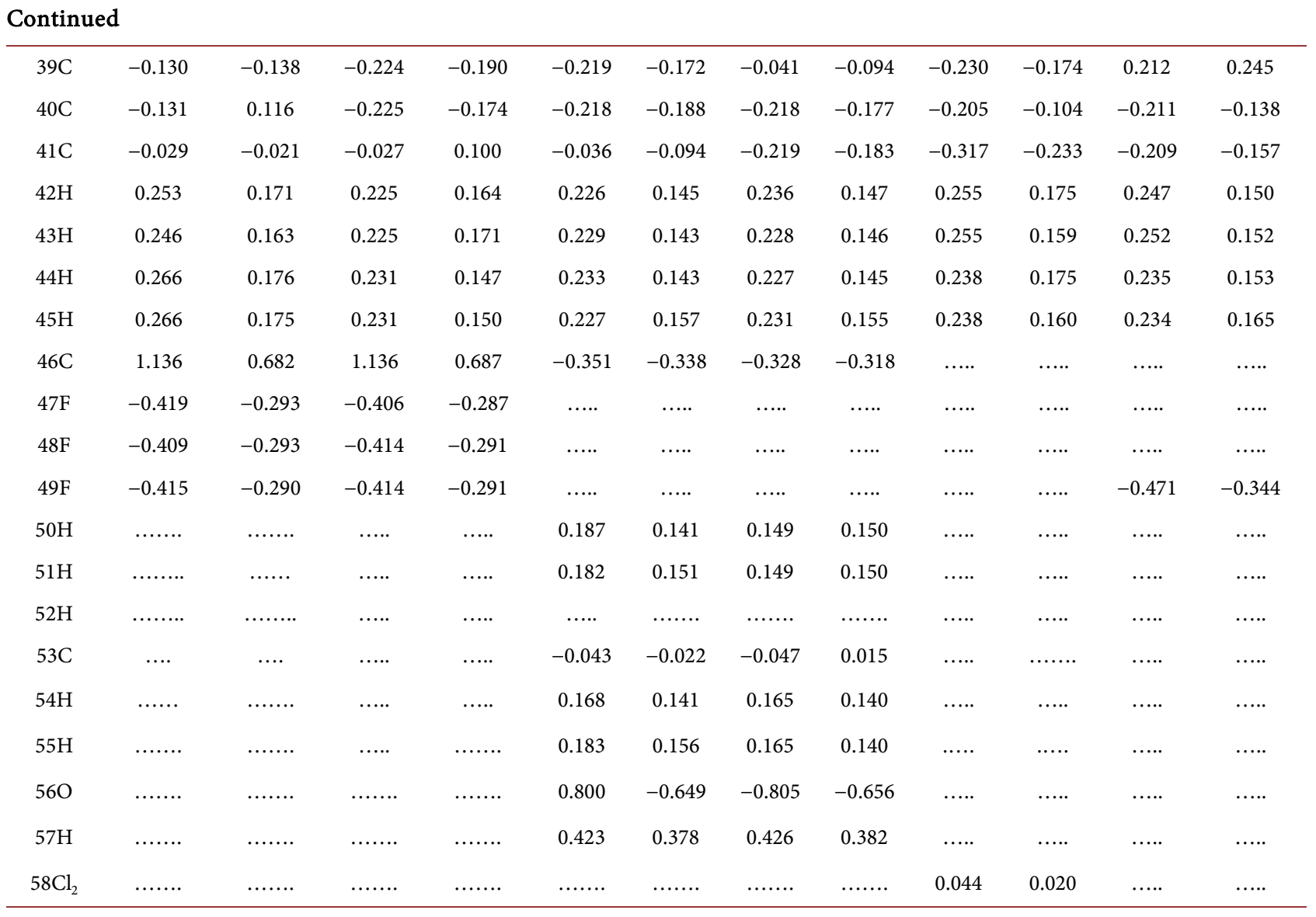

as in Table 6 and Table 7. The calculated ionization energy for the drug according to DFT and PM3 method was similar to that of Patent 2, 3, on other and was similar to Patent 5 as AM1 and HF indicated.

\subsection{Electron Affinity}

The energy of electron affinity is given by the equation: EA = -ELUMO [15]. The high value of the energy of electronic affinity means high stability and thus leads to less efficiency of the molecule to link; the low energy electronic affinity means high efficiency and less stability of a molecule. The energy of electronic affinity of the drug fluoxetine and its derivatives studied theoretically by using program G09W. The result of the electron affinity shows that Patent 3 was a comparable value with a drug in methods (HF, DFT) showed relatively similar value for the affinity between the drug and Patent 3, 4 .

\subsection{Vibration Analysis}

The observed and calculated frequencies using four methods (AM1, PM3, HF/6-31G, DFT/B3LYP/6-31G) with their absolute intensities were shown in Table 10. In order to facilitate assignment of the observed peaks we have analyzed some vibrational frequencies and compared our calculated results of the fluoxetine with their five derivatives shown in Table 10 and Figure 4. Figure 5 

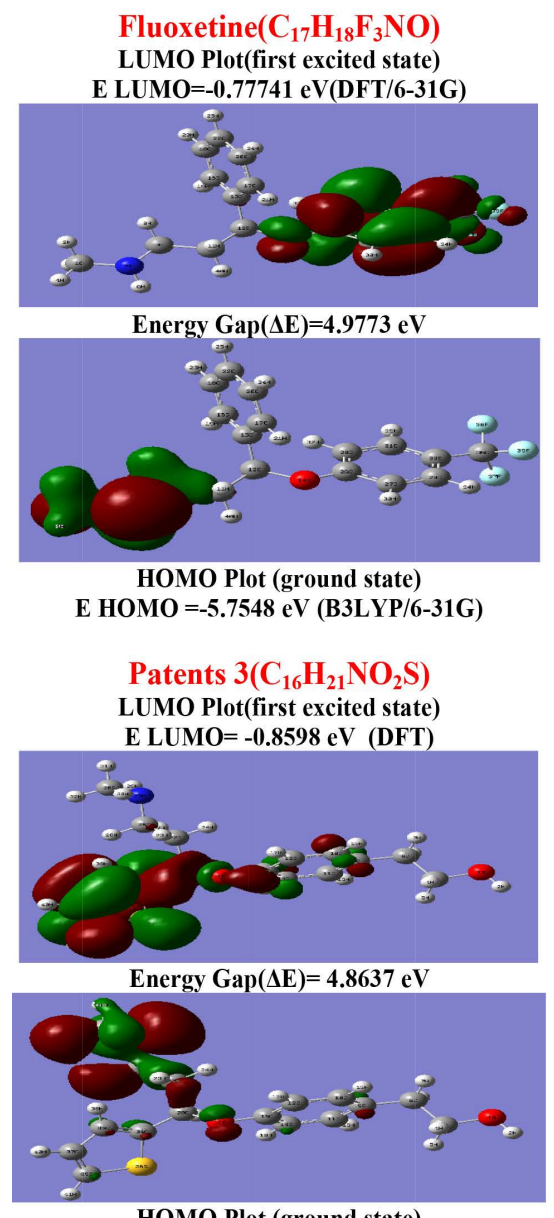

HOMO Plot (ground state) E HOMO $=-5.7235 \mathrm{eV}(\mathrm{B} 3 \mathrm{LYP} / 6-31 \mathrm{G})$

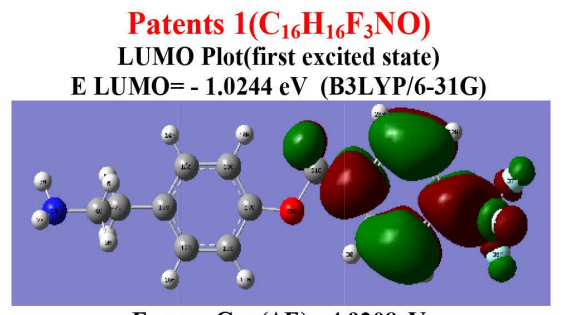

Energy $\operatorname{Gap}(\Delta E)=4.9208 \mathrm{eV}$

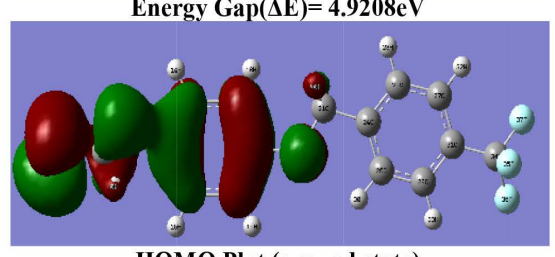

HOMO Plot (ground state)

E HOMO $=-5.9453 \mathrm{eV}(\mathrm{B3LYP} / 6-31 \mathrm{G}$

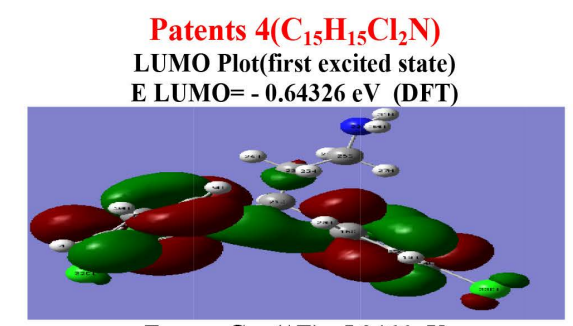

$\operatorname{Energy~} \operatorname{Gap}(\Delta \mathrm{E})=5.3466 \mathrm{eV}$

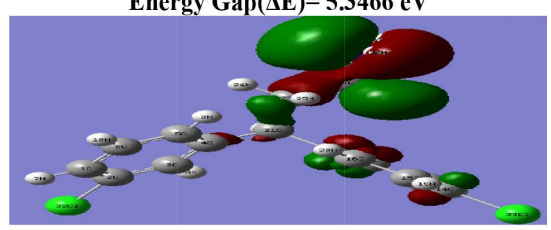

HOMO Plot (ground state) E HOMO =- 5.9899 eV (B3LYP/6-31G)
Patents $2\left(\mathrm{C}_{18} \mathrm{H}_{23} \mathrm{NO}\right)$

LUMO Plot(first excited state)

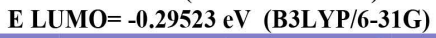

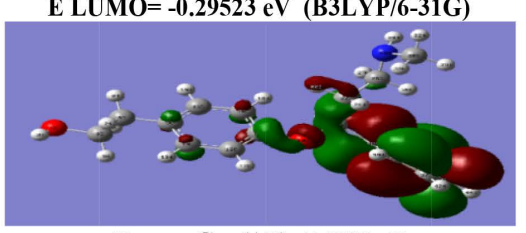

Energy $\operatorname{Gap}(\Delta E)=5.4038 \mathrm{eV}$

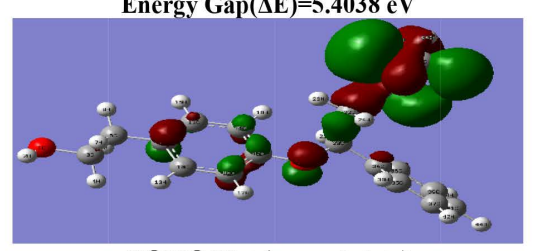

HOMO Plot (ground state)

E HOMO =- 5.6990 eV (B3LYP/6-31G)

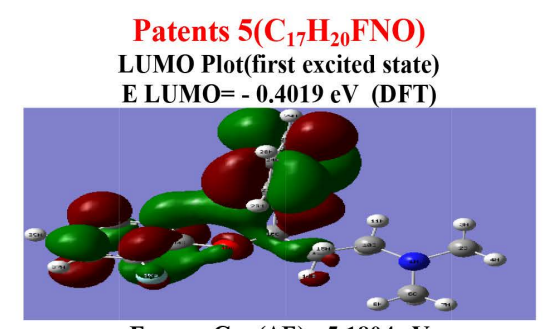

Energy $\operatorname{Gap}(\Delta \mathrm{E})=5.1804 \mathrm{eV}$

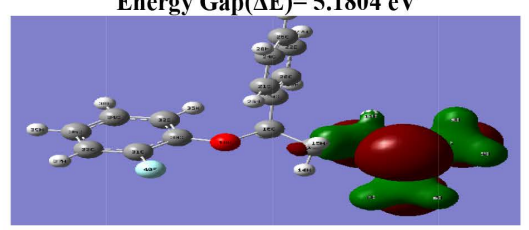

HOMO Plot (ground state)

E HOMO =- 5.5823 eV (B3LYP/6-31G)

Figure 3. Calculated HOMO-LUMO plots of drug and its derivatives using DFT/B3LYP/6-31G.

shows IR-spectra of the fluoxetine and its derivatives in four method (AM1, PM3, HF, DFT). The present study, theoretical calculations of vibrational spectra using different methods and different basis sets were compared drugs with the derivatives to obtain the best expectation. The best frequencies calculated by DFT which was in a good agreement with drug frequencies results.

\subsubsection{C-H Vibrations}

The assignment of carbon-hydrogen stretching mode is straightforward on the basis set of the scaled four methods predicted frequencies as well known group frequencies. The heteroaromatic structure shows the presence of $\mathrm{C}-\mathrm{H}$ stretching vibrations in the region $3150-3050 \mathrm{~cm}^{-1}$ [16] [17].

Which is the characteristic region for ready identification of $\mathrm{C}-\mathrm{H}$ stretching vibration? In this region, the bands are affected appreciably by the nature of the substituents. In the present theoretical study, there are three $\mathrm{C}-\mathrm{H}$ stretching vibrations observed in the aromatic ring [16] [17]. The strong and medium bands observed in IR spectrum at $(3190,3070,3434$, and 3263) in four methods for drugs are assigned to $\mathrm{C}-\mathrm{H}$ stretching vibrations as reported with the derivatives. 
Q. M. A. H. AL-Makhzumi et al.

Table 10. Comparison between experimental and theoretical values of the patterns of seismic movement frequencies drug fluoxetine and its derivatives when the geometry of the equilibrium on according to method of calculation (AM1, PM3, HF, DFT).

\begin{tabular}{|c|c|c|c|c|c|c|c|c|c|}
\hline \multicolumn{10}{|c|}{ Fluoxetine } \\
\hline & \multirow{2}{*}{$\begin{array}{c}\text { Experimental [13] } \\
\text { Freq } \cdot \mathrm{cm}^{-1}\end{array}$} & \multicolumn{2}{|c|}{ AM1 } & \multicolumn{2}{|c|}{ PM3 } & \multicolumn{2}{|c|}{$\mathrm{HF}$} & \multicolumn{2}{|c|}{ DFT } \\
\hline & & Freq $\cdot \mathrm{cm}^{-1}$ & Intensity & Freq $\cdot \mathrm{cm}^{-1}$ & Intensity & Freq $\cdot \mathrm{cm}^{-1}$ & Intensity & Freq $\cdot \mathrm{cm}^{-1}$ & Intensity \\
\hline $\mathrm{N}-\mathrm{H}$ & $3300-3500$ & 3395 & 23.31 & 3361 & 3.84 & 3772 & 31.36 & 3502 & 31.02 \\
\hline $\mathrm{C}-\mathrm{H}_{\text {aromatic }}$ & $3150-3050$ & 3190 & 96.40 & 3073 & 62.75 & 3434 & 6.83 & 3263 & 5.97 \\
\hline $\mathrm{C}-\mathrm{C}$ & $1400-1650$ & 1540 & 405.3 & 1468 & 570.3 & 1401 & 442.9 & 1339 & 447.4 \\
\hline $\mathrm{C}-\mathrm{N}$ & & 1358 & 4.11 & 1334 & 11.6 & 1298 & 2.77 & 1268 & 84.58 \\
\hline C-F & 1250 & 1278 & 127.7 & 1208 & 58.94 & 1285 & 181.9 & 1114 & 226.2 \\
\hline \multicolumn{10}{|c|}{ Patent 1} \\
\hline & Experimental [13] & \multicolumn{2}{|c|}{ AM1 } & \multicolumn{2}{|c|}{ PM3 } & \multicolumn{2}{|c|}{$\mathrm{HF}$} & \multicolumn{2}{|c|}{ DFT } \\
\hline & Freq $\cdot \mathrm{cm}^{-1}$ & Freq. $\mathrm{cm}^{-1}$ & Intensity & Freq. $\mathrm{cm}^{-1}$ & Intensity & Freq $\cdot \mathrm{cm}^{-1}$ & Intensity & Freq $\cdot \mathrm{cm}^{-1}$ & Intensity \\
\hline $\mathrm{N}-\mathrm{H}$ & $3300-3500$ & 3472 & 18.17 & 3517 & 7.40 & 3859 & 137.5 & 3609 & 167.18 \\
\hline $\mathrm{C}-\mathrm{H}_{\text {aromatic }}$ & $3150-3050$ & 3402 & 215.8 & 3065 & 55.15 & 3402 & 43.39 & 3239 & 14.15 \\
\hline $\mathrm{C}-\mathrm{C}$ & $1400-1650$ & 1321 & 318.3 & 1462 & 86.82 & 1458 & 468.1 & 1336 & 500.06 \\
\hline $\mathrm{C}-\mathrm{N}$ & $1358-1130$ & 1331 & 3.37 & 1367 & 44.98 & 1350 & 6.16 & 1430 & 23.63 \\
\hline $\mathrm{C}-\mathrm{F}$ & 1250 & 1321 & 318.3 & 1371 & 168.43 & 1245 & 373.06 & 1117 & 221.78 \\
\hline \multicolumn{10}{|c|}{ Patent 2} \\
\hline & Experimental [13] & \multicolumn{2}{|c|}{ AM1 } & \multicolumn{2}{|c|}{ PM3 } & \multicolumn{2}{|c|}{$\mathrm{HF}$} & \multicolumn{2}{|c|}{ DFT } \\
\hline & Freq $\cdot \mathrm{cm}^{-1}$ & Freq $\cdot \mathrm{cm}^{-1}$ & Intensity & Freq $\cdot \mathrm{cm}^{-1}$ & Intensity & Freq $\cdot \mathrm{cm}^{-1}$ & Intensity & Freq $\cdot \mathrm{cm}^{-1}$ & Intensity \\
\hline $\mathrm{N}-\mathrm{H}$ & $3300-3500$ & 3393 & 29.21 & 3373 & 4.32 & 3795 & 2.77 & 3530 & 0.39 \\
\hline $\mathrm{C}-\mathrm{H}_{\text {aromatic }}$ & $3150-3050$ & 3181 & 65.67 & 3074 & 34.36 & 3392 & 18.73 & 3218 & 59.91 \\
\hline $\mathrm{C}-\mathrm{C}$ & $1400-1650$ & 1450 & 61.27 & 1376 & 75.20 & 1331 & 97.67 & 1561 & 212.04 \\
\hline $\mathrm{C}-\mathrm{N}$ & $1358-1130$ & 1465 & 22.42 & 1416 & 1.16 & 1251 & 21.49 & 1376 & 75.20 \\
\hline $\mathrm{O}-\mathrm{H}$ & $3500-3450$ & 3478 & 79.15 & 3879 & 15.69 & 4005 & 62.59 & 3633 & 16.69 \\
\hline \multicolumn{10}{|c|}{ Patent 3} \\
\hline & Experimental [13] & \multicolumn{2}{|c|}{ AM1 } & \multicolumn{2}{|c|}{ PM3 } & \multicolumn{2}{|c|}{$\mathrm{HF}$} & \multicolumn{2}{|c|}{ DFT } \\
\hline & Freq $\cdot \mathrm{cm}^{-1}$ & Freq. $\mathrm{cm}^{-1}$ & Intensity & Freq $\cdot \mathrm{cm}^{-1}$ & Intensity & Freq $\cdot \mathrm{cm}^{-1}$ & Intensity & Freq $\cdot \mathrm{cm}^{-1}$ & Intensity \\
\hline $\mathrm{N}-\mathrm{H}$ & $3300-3500$ & 3394 & 28.81 & 3373 & 4.36 & 3798 & 3.36 & 3532 & 0.33 \\
\hline C- $\mathrm{H}_{\text {aromatic }}$ & $3150-3050$ & 3242 & 161.49 & 3125 & 116.3 & 3359 & 29.38 & 3195 & 33.91 \\
\hline $\mathrm{C}-\mathrm{C}$ & $1400-1650$ & 1670 & 177.4 & 1431 & 32.96 & 1691 & 179.6 & 1558 & 152.25 \\
\hline $\mathrm{C}-\mathrm{N}$ & $1358-1130$ & 1403 & 6.91 & 1415 & 1.01 & 1380 & 8.25 & 1268 & 7.17 \\
\hline $\mathrm{O}-\mathrm{H}$ & $3500-3450$ & 3482 & 97.55 & 3899 & 23.27 & 4014 & 63.29 & 3648 & 11.78 \\
\hline \multicolumn{10}{|c|}{ Patent 4} \\
\hline & Experimental [13] & \multicolumn{2}{|c|}{ AM1 } & \multicolumn{2}{|c|}{ PM3 } & & & & \\
\hline & Freq $\cdot \mathrm{cm}^{-1}$ & Freq $\cdot \mathrm{cm}^{-1}$ & Intensity & Freq $\cdot \mathrm{cm}^{-1}$ & Intensity & Freq $\cdot \mathrm{cm}^{-1}$ & Intensity & Freq $\cdot \mathrm{cm}^{-1}$ & Intensity \\
\hline $\mathrm{N}-\mathrm{H}$ & $3300-3500$ & 3459 & 16.73 & 3516 & 7.52 & 3798 & 3.36 & 3532 & 0.33 \\
\hline $\mathrm{C}-\mathrm{H}_{\text {aromatic }}$ & $3150-3050$ & 3187 & 91.65 & 31.25 & 116.3 & 3359 & 29.38 & 3195 & 33.91 \\
\hline $\mathrm{C}-\mathrm{C}$ & $1400-1650$ & 1620 & 69.78 & 1431 & 32.96 & 1691 & 179.6 & 1558 & 152.25 \\
\hline $\mathrm{C}-\mathrm{N}$ & $1358-1130$ & 1479 & 12.62 & 1415 & 1.01 & 1380 & 8.25 & 1268 & 7.17 \\
\hline $\mathrm{C}-\mathrm{Cl}$ & & 3482 & 97.55 & 3899 & 23.27 & 4014 & 63.29 & 3648 & 11.78 \\
\hline
\end{tabular}




\section{Continued}

Patent 5

\begin{tabular}{|c|c|c|c|c|c|c|c|c|c|}
\hline & \multirow{2}{*}{$\frac{\text { Experimental [13] }}{\text { Freq } \cdot \mathrm{cm}^{-1}}$} & \multicolumn{2}{|c|}{ AM1 } & \multicolumn{2}{|c|}{ PM3 } & \multicolumn{2}{|c|}{$\mathrm{HF}$} & \multicolumn{2}{|c|}{ DFT } \\
\hline & & Freq. $\mathrm{cm}^{-1}$ & Intensity & Freq. $\mathrm{cm}^{-1}$ & Intensity & Freq. $\mathrm{cm}^{-1}$ & Intensity & Freq. $\mathrm{cm}^{-1}$ & Intensity \\
\hline C- $\mathrm{H}_{\text {aromatic }}$ & $3150-3050$ & 3190 & 54.16 & 3072 & 69.66 & 3386 & 44.27 & 3229 & 25.11 \\
\hline C-C & $1400-1650$ & 1691 & 227.25 & 1684 & 191.61 & 1691 & 179.6 & 1549 & 197.89 \\
\hline $\mathrm{C}-\mathrm{N}$ & $1358-1130$ & 1497 & 25.04 & 1393 & 5.78 & 1429 & 1.81 & 1306 & 6.79 \\
\hline
\end{tabular}
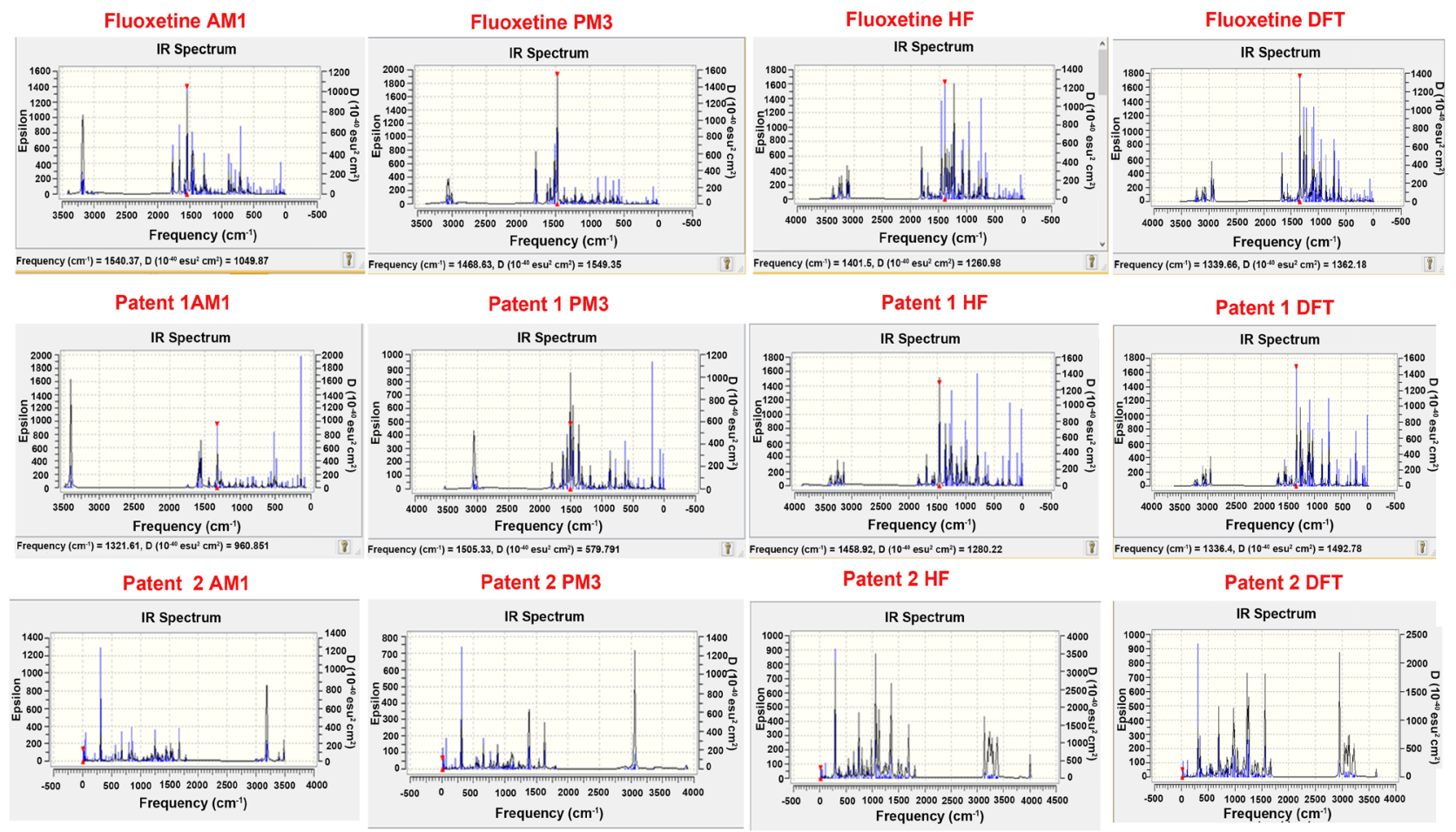

Figure 4. Calculated theoretical IR-spectra of the fluoxetine and its derivatives in four method (AM1, PM3, HF, DFT).

\subsubsection{C-C Vibrations}

There are six equivalent $\mathrm{C}-\mathrm{C}$ bonds in benzene and consequently, there will be six C-C stretching vibrations. Due to the high symmetry of benzene, many modes of vibrations are infrared inactive. In general, the bands around 1400 $1650 \mathrm{~cm}^{-1}$ in drug are assigned to skeletal stretching C-C bands [16] [17]. The bands observed at $(1540,1468,1401$, and 1339) in (AM1, PM3, HF, DFT) assigned to $\mathrm{C}-\mathrm{C}$ stretching vibrations as reported with the derivatives.

\subsubsection{C-F Vibrations}

Infrared spectra of a number of tri-fluoro group have been studied, they assigned the frequency $1250 \mathrm{~cm}^{-1}$ to a C-F stretching mode of vibration [18]. The C-F stretching vibration observed at $(1278,1208,1285$, and 1114) in (AM1, $\mathrm{PM} 3, \mathrm{HF}, \mathrm{DFT}$ ). In the present case, a band assigned to C-F stretching vibration is assigned at $1250 \mathrm{~cm}^{-1}$ according to the reported values [19]. 

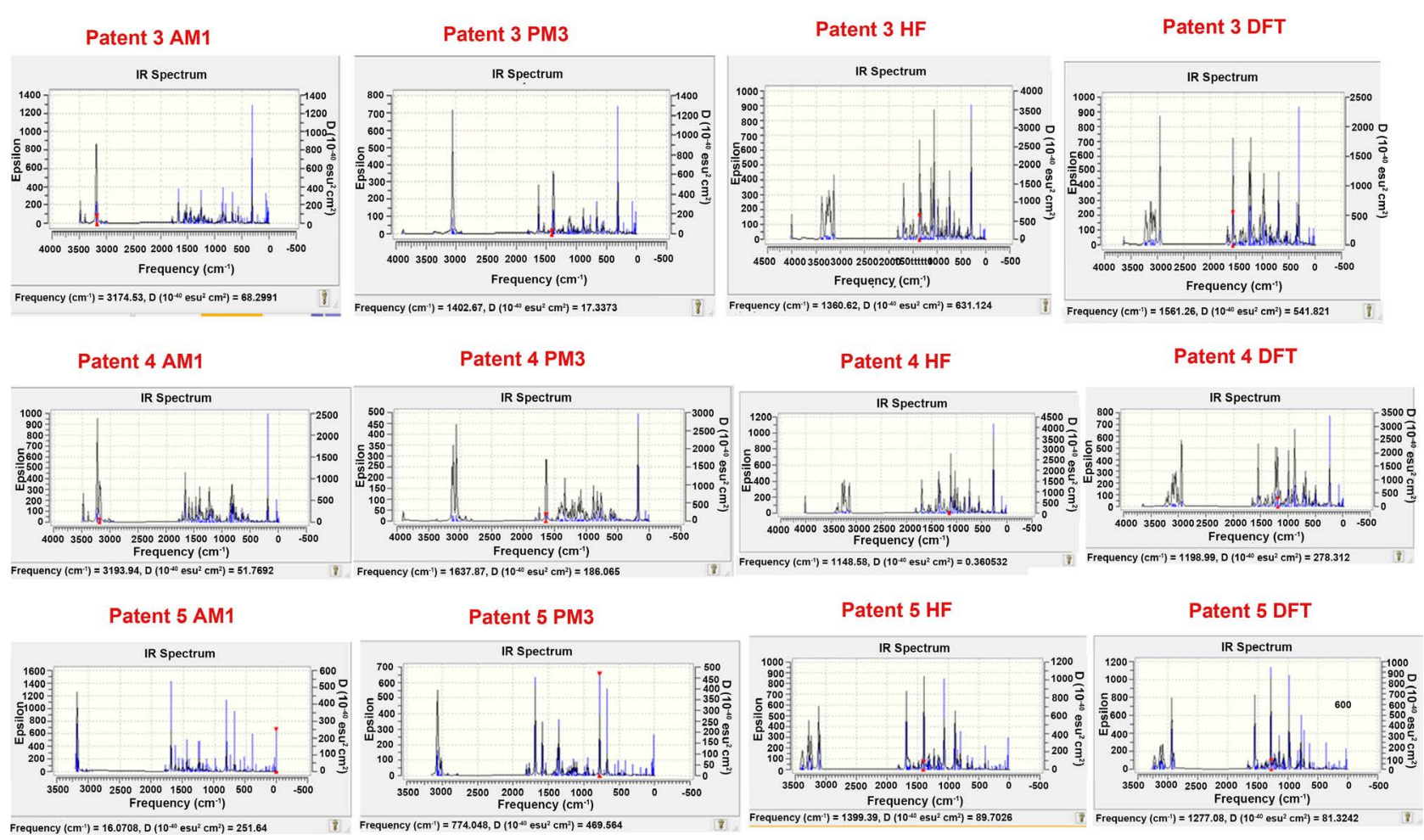

Figure 5. Calculated theoretical IR-spectra of the fluoxetine and its derivatives in four method (AM1, PM3, HF, DFT).

\subsubsection{N-H Vibrations}

Stretching type vibrations of amine functional group have $3300-3500 \mathrm{~cm}^{-1}$ characteristic IR absorption frequencies [13]. N-H stretching modes have been calculated as (3395AM1, 3361PM3, 3772HF 3502DFT) $\mathrm{cm}^{-1}$. Which are assigned the $\mathrm{N}-\mathrm{H}$ stretching modes to the frequency of drug with the derivatives? The $\mathrm{NH}$-stretching wave number in IR spectrum as weak intensity from the computed wave number indicates the weakening of the N-H bond resulting in proton transfer.

\subsection{Molecular Electrostatic Potential}

DFT method was applied to predict the molecular electrostatic potential surface of the drug and its derivatives. Figure 6 shows that the electrostatic potential as a range of color from blue to red relation to the compound activity, blue colure point to lower activity and red $\mathrm{O}$ atom to higher activity. Fluoxetine shows a higher stability (blue color) with an active tri-fluo ring group, on other hand $\mathrm{Pa}$ tents 3,4 is the most similar activity character to the drug which show a stable compound with an active group (yellow color). Patent 3, 4 shows a yellow color in zone of $\mathrm{OH}$ and Chloro group respectively. Patent 1,2, 5 shows a different active group apart from drug.

\section{Conclusions}

Theoretical studies were conducted using (AM1, PM3, HF, and DFT) methods to calculate the physical and chemical properties of fluoxetine and its five 


\section{Fluoxetine}

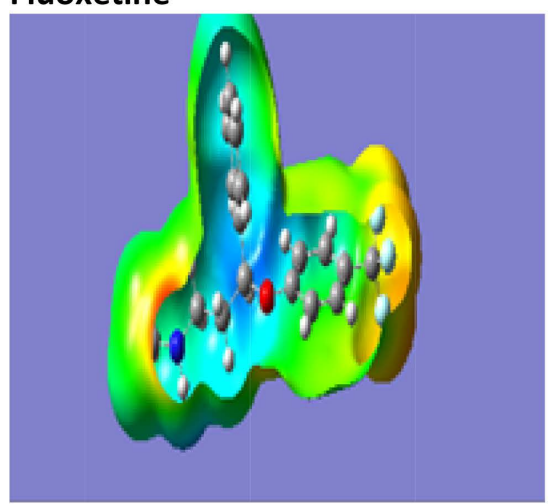

Patents 3

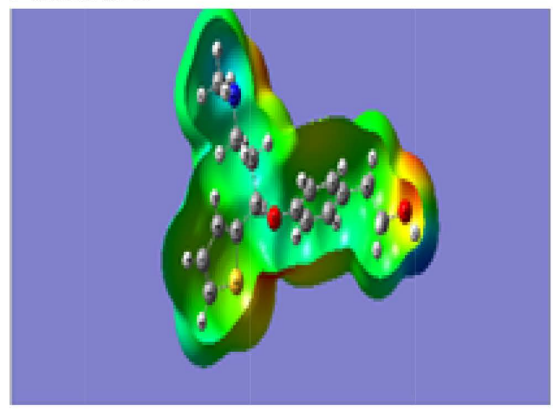

\section{Patents 1}

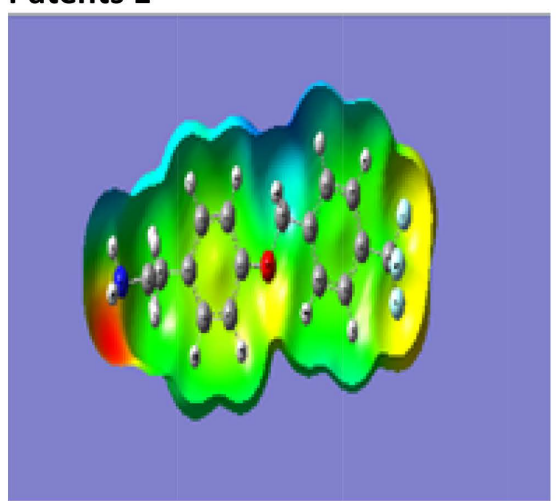

\section{Patents 4}

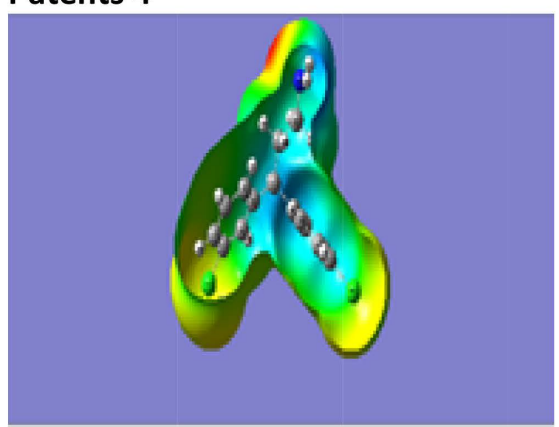

Patents 2

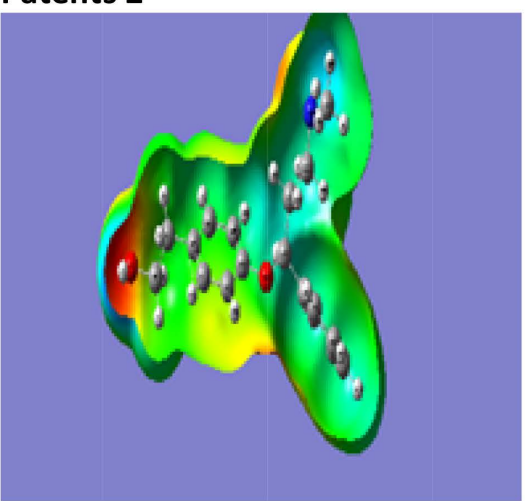

Patents 5

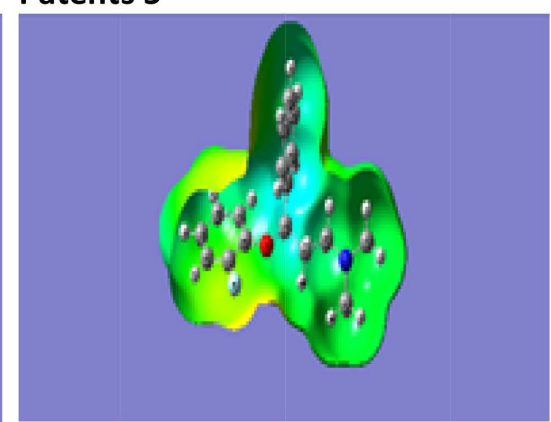

Figure 6. Molecular electrostatic potential surface of drug and its derivatives in DFT method.

derivatives as a patent, but its biological activity has not been studied.

To make an evaluation for derivatives as a drug, the energies, dipole moment, static charge, bond length, HOMO-LUMO energies and a spectrum was calculated for the optimum structure. The results of the four methods were not very clear, but a correlation between the dipole moment (potential character), static distribution (an active site character) and HOMO-LUMO energies (energy for electron transfer) show that the Patent 1, 5 was important derivatives as a recommended drug relative to fluoxetine drug.

\section{Conflicts of Interest}

The authors declare no conflicts of interest regarding the publication of this paper.

\section{References}

[1] Mostert, J.P., Koch, M.W., Heerings, M., Heersema, D.J. and De Keyser, J. (2008) Therapeutic Potential of Fluoxetine in Neurological Disorders. CNS Neuroscience \& Therapeutics, 14, 153-164. https://doi.org/10.1111/j.1527-3458.2008.00040.x

[2] Richman, A. and Heinrichs, S.C. (2007) Seizure Prophylaxis in an Animal Model of Epilepsy by Dietary Fluoxetine Supplementation, Epilepsy Research, 74, 19-27. https://doi.org/10.1016/j.eplepsyres.2006.11.007

[3] Choudhary, N., Bee, S., Gupta, A. and Tandon, P. (2013) Comparative Vibrational Spectroscopic Studies, HOMO-LUMO and NBO Analysis of N-(Phenyl)-2,2-Dic- 
Hloroacetamide, N-(2-Chloro Phenyl)-2,2-Dichloroacetamide and N-(4-Chloro Phenyl)-2,2-Dichloroacetamide Based on Density Functional Theory. Computational and Theoretical Chemistry, 1016, 8-21. https://doi.org/10.1016/j.comptc.2013.04.008

[4] Valeur, E. and Bradley, M. (2009) Amide Bond Formation: Beyond the Myth of Coupling Reagents. Chemical Society Reviews, 38, 606-631.

https://doi.org/10.1039/B701677H

[5] Patchett, A.A. (1993) Excursions in Drug Discovery. Journal of Medicinal Chemistry, 36, 2051-2058. https://doi.org/10.1021/jm00067a001

[6] Tomberg, A. (2013) Gaussian 09W Tutorial, an Introduction to Computational Chemistry Using G09W and Avogadro Software. 1-34.

[7] Barone, V., Bloino, J. and Biczysko, M. (2009) Vibrationally-Resolved Electronic spectra in Gaussian 09. Revision a2.

[8] Carter, C.K. and Kohn, R. (1996) Markov Chain Monte Carlo in Conditionally Gaussian State Space Models. Biometrika, 83, 589-601.

[9] Runge, E. and Gross, E.K. (1984) Density-Functional Theory for Time-Dependent Systems. Physical Review Letters, 52, 997. https://doi.org/10.1103/PhysRevLett.52.997

[10] Karunakaran, V. and Balachandran, V. (2014) Experimental and Theoretical Investigation of the Molecular Structure, Conformational Stability, Hyperpolarizability, Electrostatic Potential, Thermodynamic Properties and NMR Spectra of Pharmaceutical Important Molecule: 4'-Methylpropiophenone. Spectrochimica Acta Part A: Molecular and Biomolecular Spectroscopy, 128, 1-14. https://doi.org/10.1016/j.saa.2014.02.155

[11] Çırak, Ç., Sert, Y. and Ucun, F. (2012) Experimental and Computational Study on Molecular Structure and Vibrational Analysis of a Modified Biomolecule: 5-Bromo-2'-Deoxyuridine. Spectrochimica Acta Part A: Molecular and Biomolecular Spectroscopy, 92, 406-414. https://doi.org/10.1016/j.saa.2012.02.053

[12] Xavier, R.J. and Gobinath, E. (2012) Density Functional Theory Study on Characterization of 3-Chloro-1,2-Benzisothiazole. Spectrochimica Acta Part A: Molecular and Biomolecular Spectroscopy, 91, 248-255. https://doi.org/10.1016/j.saa.2012.01.083

[13] Kwon, J. (2012) Applications of Semi-Empirical Quantum Mechanical Calculations to Simple Molecular Systems. Emporia State University, Emporia.

[14] Gross, J.H. (2017) Principles of Ionization and Ion Dissociation, In: Mass Spectrometry, 29-84, Springer, Cham. https://doi.org/10.1007/978-3-319-54398-7_2

[15] McNaught, A.D. and McNaught, A.D. (1997) Compendium of Chemical Terminology. Vol. 1669, Blackwell Science Ltd., Oxford.

[16] Arivazhagan, M. and Rexalin, D.A. (2012) FT-IR, FT-Raman, NMR Studies and ab Initio-HF, DFT-B3LYP Vibrational Analysis of 4-Chloro-2-Fluoroaniline. Spectrochimica Acta Part A: Molecular and Biomolecular Spectroscopy, 96, 668-676. https://doi.org/10.1016/j.saa.2012.07.040

[17] Arivazhagan, M. and Jeyavijayan, S. (2011) FTIR and FT-Raman Spectra, Assignments, ab Initio HF and DFT Analysis of Xanthine. Spectrochimica Acta Part A: Molecular and Biomolecular Spectroscopy, 79, 161-168. https://doi.org/10.1016/j.saa.2011.02.029

[18] Rastogi, V., Palafox, M.A., Tanwar, R. and Mittal, L. (2002) 3,5-Difluorobenzonitrile: ab Initio Calculations, FTIR and Raman Spectra. Spectrochimica Acta Part A: Mo- 
lecular and Biomolecular Spectroscopy, 58, 1987-2004.

https://doi.org/10.1016/S1386-1425(01)00650-3

[19] Sundaraganesan, N., Meganathan, C., Joshua, B.D., Mani, P. and Jayaprakash, A. (2008) Molecular Structure and Vibrational Spectra of 3-Chloro-4-Fluoro Benzonitrile by ab Initio HF and Density Functional Method. Spectrochimica Acta Part A: Molecular and Biomolecular Spectroscopy, 71, 1134-1139.

https://doi.org/10.1016/j.saa.2008.03.019 